\title{
Multiple Weak C-H Intramolecular Hydrogen Bonding as an Aid to Minimizing Bond Rotation Flexibility
}

John Andrew Harrison,* Muhammad Arif Sajjad, Peter Schwerdtfeger and Alastair (Al) James Nielson*

\section{Supplementary Data}

Supplementary Data Table S1: X-Ray crystallographic and DFT functional comparison of important bond distances $(\AA)$, bond angles $\left(^{\circ}\right)$ and separations $(\AA)$.

Supplementary Data Table S2: NBO donor-acceptor properties for energy minimised structure and the single point calculation of the X-ray structure.

Supplementary Data Table S3: Close contact distances $(\AA)$ and NCI iso-surface colour comparisons for the calculated X-ray (X-rayC) and PBE-D3 energy minimised (Emin) structures.

Supplementary Data Table S4: Close contact distances $(\AA)$ and bond critical point (bcp) data comparisons for the calculated $\mathrm{X}$-ray $(\mathrm{X}$-rayC) and $\mathrm{PBE}-\mathrm{D} 3$ energy minimised (Emin) structures.

Supplementary Data Table S5: Atomic charge $[q(\mathrm{~A}), q(\mathrm{~B})$ partner] comparisons for the calculated X-ray (X-rayC) and PBE-D3 energy minimised (Emin)structures.

Supplementary Data Table S6: Cartesian coordinates and total energies for the energy minimised and single point calculation versions of $\left[\mathrm{Mo}\left(\mathrm{NC}_{6} \mathrm{H}_{4} \mathrm{CMe}_{3}-2\right)(\mathrm{O}) \mathrm{Cl}_{2}\right.$ (bipy)].

Supplementary Date Table S7: Comparison of structural and QTAIM parameters of Energy minimised (Emin), X-ray (xray), and the complexes (a-d), structures.

Supplementary Figures S1-S10: Comparison of energy minimised (EM) and single point (SP) iso-surfaces.

Supplementary Figure S11: Molecular graphs for the calculated X-ray (X-rayC) and PBE-D3 energy minimised (Emin) structures.

Supplementary Figure S12: Unit cell obtained from the X-ray crystal structure.

Supplementary Figure S13: Molecular graphs showing intermolecular bond critical points with dicholoromethane solvent molecules for complexes a-d.

Supplementary Figure S14: Geometries of the complexes (a-d) containing $\mathrm{CH}_{2} \mathrm{Cl}_{2}$. 
Supplementary Data Table S1: X-Ray crystallographic and DFT functional comparison of important bond distances $(\AA)$, bond angles $\left(^{\circ}\right)$ and separations $(\AA)$.

\begin{tabular}{|l|l|l|l|c|l|l|l|c|}
\hline Parameter & X-Ray & $3 \sigma$ & PBE-D3 & diff & BP86 & diff & B3LYP & diff \\
\hline $\mathrm{Mo}=\mathrm{O}(1)$ & $1.686(2)$ & 1.692 & 1.714 & 0.022 & 1.716 & 0.024 & 1.697 & 0.005 \\
\hline $\mathrm{Mo}=\mathrm{N}(1)$ & $1.735(3)$ & 1.744 & 1.771 & 0.027 & 1.777 & 0.033 & 1.758 & 0.014 \\
\hline $\mathrm{Mo}-\mathrm{N}(2)$ & $2.255(3)$ & 2.264 & 2.377 & 0.113 & 2.338 & 0.074 & 2.356 & 0.092 \\
\hline $\mathrm{Mo}-\mathrm{N}(3)$ & $2.283(3)$ & 2.292 & 2.406 & 0.114 & 2.387 & 0.095 & 2.403 & 0.111 \\
\hline $\mathrm{Mo}-\mathrm{Cl}(1)$ & $2.358(1)$ & 2.361 & 2.388 & 0.027 & 2.393 & 0.032 & 2.406 & 0.045 \\
\hline $\mathrm{Mo}-\mathrm{Cl}(2)$ & $2.375(1)$ & 2.378 & 2.406 & 0.028 & 2.409 & 0.031 & 2.419 & 0.041 \\
\hline & & & & & & & & \\
\hline $\mathrm{Cl}-\mathrm{Mo}-\mathrm{Cl}$ & $160.08(3)$ & 160.0 & 154.1 & 5.9 & 154.5 & 5.5 & 154.8 & 5.2 \\
\hline $\mathrm{O}(1)-\mathrm{Mo}-\mathrm{Cl}(1)$ & $96.8(1)$ & 97.1 & 98.6 & 1.5 & 98.5 & 1.4 & 98.2 & 1.1 \\
\hline $\mathrm{O}(1)-\mathrm{Mo}-\mathrm{Cl}(2)$ & $97.4(1)$ & 97.7 & 99.5 & 1.8 & 99.3 & 1.6 & 99.4 & 1.7 \\
\hline $\mathrm{O}(1)-\mathrm{Mo}-\mathrm{N}(1)$ & $104.7(1)$ & 105.0 & 106.0 & 1.0 & 105.6 & 0.6 & 105.5 & 0.5 \\
\hline $\mathrm{O}(1)-\mathrm{Mo}-\mathrm{N}(2)$ & $89.3(1)$ & 89.6 & 89.6 & 0 & 88.4 & 1.2 & 88.4 & 1.2 \\
\hline $\mathrm{O}(1)-\mathrm{Mo}-\mathrm{N}(3)$ & $159.2(1)$ & 158.9 & 158.5 & 0.4 & 157.2 & 1.7 & 156.6 & -2.3 \\
\hline $\mathrm{N}(1)-\mathrm{Mo}-\mathrm{N}(2)$ & $164.4(1)$ & 164.1 & 163.7 & 0.4 & 165.2 & -0.5 & 165.3 & 1.2 \\
\hline $\mathrm{N}(1)-\mathrm{Mo}-\mathrm{N}(3)$ & $96.0(1)$ & 95.7 & 95.5 & 0.2 & 97.2 & -1.1 & 97.9 & -1.6 \\
\hline $\mathrm{N}(2)-\mathrm{Mo}-\mathrm{N}(3)$ & $70.0(1)$ & 69.7 & 69.0 & 0.7 & 68.8 & 0.9 & 68.2 & 1.5 \\
\hline $\mathrm{Mo}-\mathrm{N}(1)-\mathrm{C}(11)$ & $165.8(2)$ & 166.4 & 166.7 & 0.3 & 167.4 & 1.0 & 168.0 & 1.6 \\
\hline
\end{tabular}

Supplementary Data Table S2: NBO donor-acceptor properties for energy minimised structure and the single point calculation of the X-ray structure.

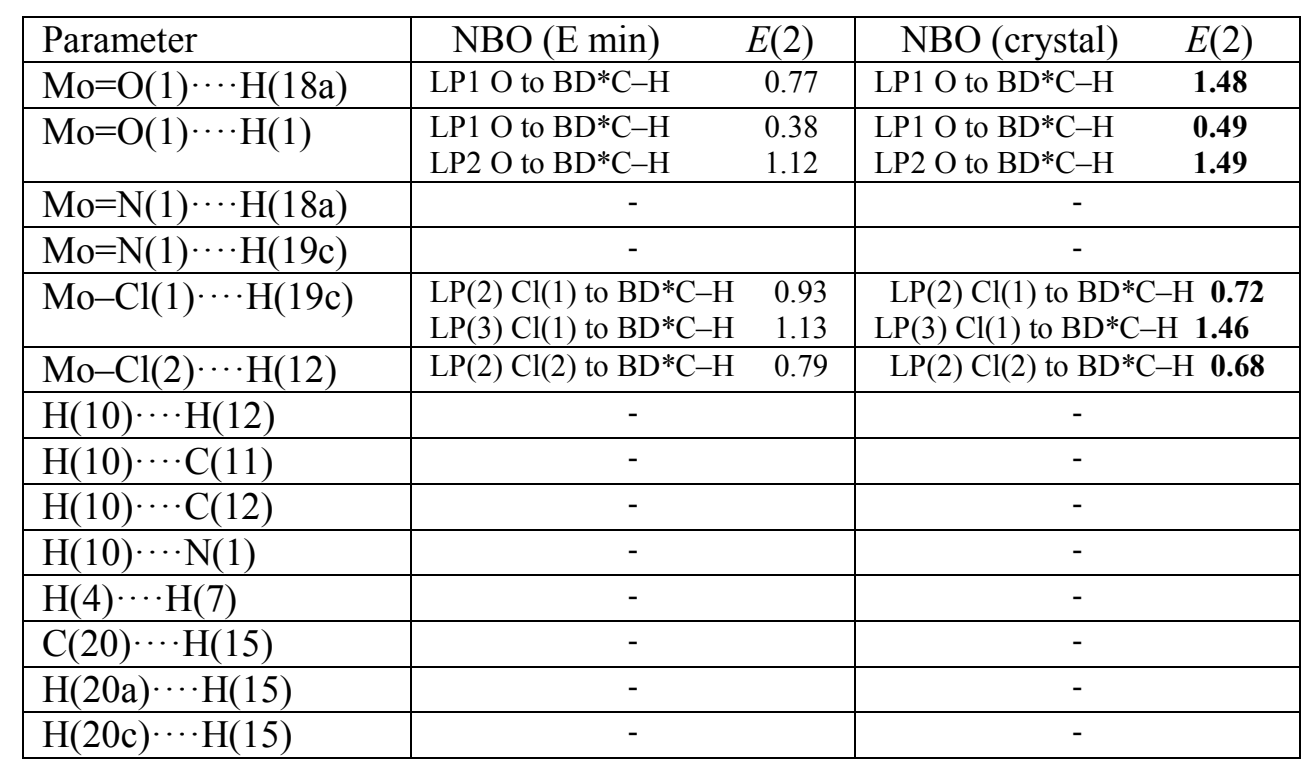


Supplementary Data Table S3: Close contact distances $(\AA)$ and NCI iso-surface colour comparisons for the calculated X-ray (X-rayC) and PBE-D3 energy minimised (Emin) structures.

\begin{tabular}{|l|c|c|c|c|c|}
\hline Parameter & $\begin{array}{c}\text { X-rayC } \\
\text { distance }\end{array}$ & $\begin{array}{c}\text { Emin } \\
\text { distance }\end{array}$ & difference & $\begin{array}{c}\text { X-rayC } \\
\text { iso-surface }\end{array}$ & $\begin{array}{c}\text { Emin } \\
\text { iso-surface }\end{array}$ \\
\hline $\mathrm{Mo}=\mathrm{O}(1) \cdots \mathrm{H}(18 \mathrm{a})$ & $\mathbf{2 . 3 9 6}$ & 2.586 & $\mathbf{0 . 1 9 0}$ & green & green \\
\hline $\mathrm{Mo}=\mathrm{O}(1) \cdots \mathrm{H}(1)$ & $\mathbf{2 . 3 2 4}$ & 2.380 & $\mathbf{0 . 0 5 6}$ & green & Bluish green \\
\hline $\mathrm{Mo}=\mathrm{N}(1) \cdots \mathrm{H}(18 \mathrm{a})$ & $\mathbf{2 . 4 2 0}$ & 2.407 & $\mathbf{- 0 . 0 1 3}$ & Bluish green & Bluish green \\
\hline $\mathrm{Mo}=\mathrm{N}(1) \cdots \mathrm{H}(19 \mathrm{c})$ & $\mathbf{2 . 3 6 7}$ & 2.466 & $\mathbf{0 . 0 9 9}$ & Bluish green & Bluish green \\
\hline $\mathrm{Mo}-\mathrm{Cl}(1) \cdots \mathrm{H}(19 \mathrm{c})$ & $\mathbf{2 . 6 9 1}$ & 2.722 & $\mathbf{0 . 0 3 1}$ & green & Light Bluish green \\
\hline $\mathrm{Mo}-\mathrm{Cl}(2) \cdots \mathrm{H}(12)$ & $\mathbf{2 . 9 3 6}$ & 2.993 & $\mathbf{0 . 0 5 7}$ & green & green \\
\hline $\mathrm{H}(10) \cdots \mathrm{H}(12)$ & $\mathbf{2 . 4 5 9}$ & 2.511 & $\mathbf{0 . 0 5 2}$ & green & green \\
\hline $\mathrm{H}(10) \cdots \mathrm{C}(11)$ & $\mathbf{2 . 9 0 1}$ & 2.888 & $\mathbf{- 0 . 0 1 3}$ & green & green \\
\hline $\mathrm{H}(10) \cdots \mathrm{C}(12)$ & $\mathbf{2 . 7 9 9}$ & 2.780 & $\mathbf{- 0 . 0 1 9}$ & green & green \\
\hline $\mathrm{H}(10) \cdots \mathrm{N}(1)$ & $\mathbf{2 . 6 3 7}$ & 2.692 & $\mathbf{0 . 0 5 5}$ & Bluish green & Bluish green \\
\hline $\mathrm{H}(4) \cdots \mathrm{H}(7)$ & $\mathbf{2 . 0 1 8}$ & 2.063 & $\mathbf{0 . 0 4 5}$ & Bluish green & Bluish green \\
\hline $\mathrm{C}(20)^{\cdots} \cdot \mathrm{H}(15)$ & $\mathbf{2 . 3 8 1}$ & 2.428 & $\mathbf{0 . 0 4 7}$ & Bluish green & Bluish green \\
\hline $\mathrm{H}(20 \mathrm{a}) \cdots \mathrm{H}(15)$ & $\mathbf{2 . 1 6 0}$ & 2.190 & $\mathbf{0 . 0 3 0}$ & green & green \\
\hline $\mathrm{H}(20 \mathrm{c}) \cdots \mathrm{H}(15)$ & $\mathbf{2 . 1 7 4}$ & 2.231 & $\mathbf{0 . 0 5 7}$ & green & green \\
\hline
\end{tabular}

Supplementary Data Table S4: Close contact distances $(\AA)$ and bond critical point (bcp) data comparisons for the calculated X-ray (X-rayC) and PBE-D3 energy minimised (Emin) structures.

\begin{tabular}{|l|c|c|c|c|}
\hline Parameter & X-rayC distance & X-rayC bcp & Emin distance & Emin bcp \\
\hline $\mathrm{Mo}=\mathrm{O}(1) \cdots \mathrm{H}(18 \mathrm{a})$ & $\mathbf{2 . 3 9 6}$ & yes & 2.586 & - \\
\hline $\mathrm{Mo}=\mathrm{O}(1) \cdots \mathrm{H}(1)$ & $\mathbf{2 . 3 2 4}$ & yes & 2.380 & yes \\
\hline $\mathrm{Mo}=\mathrm{N}(1) \cdots \mathrm{H}(18 \mathrm{a})$ & $\mathbf{2 . 4 2 0}$ & yes & 2.407 & yes \\
\hline $\mathrm{Mo}=\mathrm{N}(1) \cdots \mathrm{H}(19 \mathrm{c})$ & $\mathbf{2 . 3 6 7}$ & yes & 2.466 & yes \\
\hline $\mathrm{Mo}-\mathrm{Cl}(1) \cdots \mathrm{H}(19 \mathrm{c})$ & $\mathbf{2 . 6 9 1}$ & yes & 2.722 & yes \\
\hline $\mathrm{Mo}-\mathrm{Cl}(2) \cdots \mathrm{H}(12)$ & $\mathbf{2 . 9 3 6}$ & yes & 2.993 & yes \\
\hline $\mathrm{H}(10) \cdots \cdot \mathrm{H}(12)$ & $\mathbf{2 . 4 5 9}$ & - & 2.511 & - \\
\hline $\mathrm{H}(10) \cdots \mathrm{C}(11)$ & $\mathbf{2 . 9 0 1}$ & - & 2.888 & - \\
\hline $\mathrm{H}(10) \cdots \mathrm{C}(12)$ & $\mathbf{2 . 7 9 9}$ & yes & 2.780 & yes \\
\hline $\mathrm{H}(10) \cdots \mathrm{N}(1)$ & $\mathbf{2 . 6 3 7}$ & - & 2.692 & - \\
\hline $\mathrm{H}(4) \cdots \cdot \mathrm{H}(7)$ & $\mathbf{2 . 0 1 8}$ & yes & 2.063 & yes \\
\hline $\mathrm{C}(20) \cdots \mathrm{H}(15)$ & $\mathbf{2 . 3 8 1}$ & - & 2.428 & - \\
\hline $\mathrm{H}(20 \mathrm{a}) \cdots \cdot \mathrm{H}(15)$ & $\mathbf{2 . 1 6 0}$ & - & 2.190 & - \\
\hline $\mathrm{H}(20 \mathrm{c}) \cdots \cdot \mathrm{H}(15)$ & $\mathbf{2 . 1 7 4}$ & - & 2.231 & - \\
\hline
\end{tabular}


Supplementary Data Table S5: Atomic charge [ $q(\mathrm{~A}), q(\mathrm{~B})$ partner] comparisons for the calculated X-ray (X-rayC) and PBE-D3 energy minimised (Emin)structures.

\begin{tabular}{|l|c|c|c|c|}
\hline Separation & $\begin{array}{c}\text { X-RayC } \\
\boldsymbol{q}(\mathbf{A})\end{array}$ & $\begin{array}{c}\text { X-RayC } \\
\boldsymbol{q}(\mathbf{B})\end{array}$ & $\begin{array}{l}\text { Emin } \\
\boldsymbol{q}(\mathbf{A})\end{array}$ & $\begin{array}{l}\text { Emin } \\
\boldsymbol{q}(\mathbf{B})\end{array}$ \\
\hline $\mathrm{Mo}=\mathrm{O}(1) \cdots \mathrm{H}(18 \mathrm{a})$ & $\mathbf{- 0 . 7 8 8 7}$ & $\mathbf{0 . 0 3 4 2}$ & -0.7796 & 0.0308 \\
\hline $\mathrm{Mo}=\mathrm{O}(1) \cdots \cdot \mathrm{H}(1)$ & $\mathbf{- 0 . 7 8 8 7}$ & $\mathbf{0 . 1 0 6 6}$ & -0.7796 & 0.1100 \\
\hline $\mathrm{Mo}=\mathrm{N}(1) \cdots \cdot \mathrm{H}(18 \mathrm{a})$ & $\mathbf{- 1 . 0 5 3 1}$ & $\mathbf{0 . 0 3 4 2}$ & -1.0455 & 0.0308 \\
\hline $\mathrm{Mo}=\mathrm{N}(1) \cdots \cdot \mathrm{H}(19 \mathrm{c})$ & $\mathbf{- 1 . 0 5 3 1}$ & $\mathbf{0 . 0 2 1 5}$ & -1.0455 & 0.0219 \\
\hline $\mathrm{Mo}-\mathrm{Cl}(1) \cdots \mathrm{H}(19 \mathrm{c})$ & $\mathbf{- 0 . 5 6 5 6}$ & $\mathbf{0 . 0 2 1 5}$ & -0.5532 & 0.0219 \\
\hline $\mathrm{Mo}-\mathrm{Cl}(2) \cdots \mathrm{H}(12)$ & $\mathbf{- 0 . 5 7 1 9}$ & $\mathbf{0 . 0 2 9 4}$ & -0.5614 & 0.0402 \\
\hline $\mathrm{H}(10) \cdots \cdot \mathrm{H}(12)$ & $\mathbf{0 . 0 7 7 1}$ & $\mathbf{0 . 0 2 9 4}$ & 0.0838 & 0.0402 \\
\hline $\mathrm{H}(10) \cdots \mathrm{C}(11)$ & $\mathbf{0 . 0 7 7 1}$ & $\mathbf{0 . 4 2 7 0}$ & 0.0838 & 0.4192 \\
\hline $\mathrm{H}(10) \cdots \mathrm{C}(12)$ & $\mathbf{0 . 0 7 7 1}$ & $\mathbf{- 0 . 0 1 4 3}$ & 0.0838 & -0.0238 \\
\hline $\mathrm{H}(10) \cdots \mathrm{N}(1)$ & $\mathbf{0 . 0 7 7 1}$ & $\mathbf{- 1 . 0 5 3 1}$ & 0.0838 & -1.0455 \\
\hline $\mathrm{H}(4) \cdots \cdot \mathrm{H}(7)$ & $\mathbf{0 . 0 2 5 2}$ & $\mathbf{0 . 0 2 6 0}$ & 0.0336 & 0.0334 \\
\hline $\mathrm{C}(20) \cdots \mathrm{H}(15)$ & $\mathbf{0 . 0 4 5 9}$ & $\mathbf{0 . 0 0 7 6}$ & 0.0443 & 0.0157 \\
\hline $\mathrm{H}(20 \mathrm{a}) \cdots \cdot \mathrm{H}(15)$ & $\mathbf{- 0 . 0 2 6 1}$ & $\mathbf{0 . 0 0 7 6}$ & -0.0223 & 0.0157 \\
\hline $\mathrm{H}(20 \mathrm{c}) \cdots \mathrm{H}(15)$ & $\mathbf{- 0 . 0 2 8 4}$ & $\mathbf{0 . 0 0 7 6}$ & -0.0253 & 0.0157 \\
\hline
\end{tabular}

Supplementary Data Table S6: Cartesian coordinates and total energies for the energy minimised and single point calculation versions of $\left[\mathrm{Mo}\left(\mathrm{NC}_{6} \mathrm{H}_{4} \mathrm{CMe}_{3}-2\right)(\mathrm{O}) \mathrm{Cl}_{2}(\right.$ bipy $\left.)\right]$.

Energy minimised structure: $(E=-2001.45808553$ a.u $)$

$\begin{array}{lrrr}0 ~ 1 & & & \\ \mathrm{Mo} & 0.33340300 & -0.73307700 & -0.25634300 \\ \mathrm{Cl} & 0.60989300 & -1.10672600 & 2.08567600 \\ \mathrm{~N} & 2.65434800 & -0.91974900 & -0.24386000 \\ \mathrm{Cl} & 0.87181400 & 0.29736100 & -2.36150700 \\ \mathrm{~N} & 1.34842200 & 1.29336000 & 0.46173600 \\ \mathrm{~N} & -1.32215900 & -0.10243500 & -0.26346600 \\ \mathrm{O} & 0.21688700 & -2.36635200 & -0.76322200 \\ \mathrm{C} & 2.70195200 & 1.37144600 & 0.49828100 \\ \mathrm{C} & 0.61127600 & 2.35397000 & 0.83931500 \\ \mathrm{C} & 3.34924700 & 2.55199400 & 0.90769100 \\ \mathrm{C} & 2.58329200 & 3.65940500 & 1.28753200 \\ \mathrm{C} & 1.18579400 & 3.55995200 & 1.25766700 \\ \mathrm{C} & 3.23369800 & -2.07956900 & -0.60756900 \\ \mathrm{C} & 3.42869100 & 0.14145300 & 0.09962400 \\ \mathrm{C} & 4.83212500 & 0.04759600 & 0.07783500 \\ \mathrm{C} & 4.62320300 & -2.24259500 & -0.65068700 \\ \mathrm{C} & 5.43600500 & -1.15585400 & -0.30231600 \\ \mathrm{C} & -2.48460300 & 0.58954000 & -0.48171100 \\ \mathrm{C} & -3.77501800 & 0.12153300 & -0.05354500 \\ \mathrm{C} & -2.36461000 & 1.81479100 & -1.19905100 \\ \mathrm{C} & -3.48929600 & 2.58255200 & -1.50652100 \\ \mathrm{C} & -4.87342200 & 0.94534700 & -0.36178700 \\ \mathrm{C} & -4.75180500 & 2.15048600 & -1.07431500 \\ \mathrm{C} & -3.94514700 & -1.20083300 & 0.71030100 \\ \mathrm{H} & 5.04990000 & -3.20595400 & -0.95428200 \\ \mathrm{H} & 2.53724600 & -2.88643600 & -0.86961500 \\ \mathrm{H} & 4.44272000 & 2.60974100 & 0.93390800 \\ \mathrm{H} & -1.36572300 & 2.10889700 & -1.54566900 \\ \mathrm{H} & -5.87645800 & 0.63713400 & -0.04694000 \\ \mathrm{H} & -5.64835500 & 2.74546600 & -1.29350100 \\ \mathrm{H} & -3.37927800 & 3.51282200 & -2.07983000 \\ \mathrm{C} & -3.41186800 & -2.37612200 & -0.14762000\end{array}$




$\begin{array}{lrrr}\mathrm{H} & -3.55688800 & -3.32693200 & 0.39926600 \\ \mathrm{H} & -3.96764800 & -2.44094000 & -1.10148200 \\ \mathrm{H} & -2.33911500 & -2.27954100 & -0.37886200 \\ \mathrm{C} & -5.42609400 & -1.49151200 & 1.02890600 \\ \mathrm{H} & -6.03849000 & -1.58152900 & 0.11199400 \\ \mathrm{H} & -5.49451100 & -2.45297300 & 1.56997100 \\ \mathrm{H} & -5.87367800 & -0.71447800 & 1.67686500 \\ \mathrm{C} & -3.18222300 & -1.12379600 & 2.05489900 \\ \mathrm{H} & -2.10213300 & -0.95639200 & 1.91454900 \\ \mathrm{H} & -3.58331800 & -0.30480200 & 2.68133100 \\ \mathrm{H} & -3.30517200 & -2.07271000 & 2.61048300 \\ \mathrm{H} & 5.45269000 & 0.90653100 & 0.35466200 \\ \mathrm{H} & 6.52998700 & -1.24205800 & -0.32502400 \\ \mathrm{H} & 3.07407700 & 4.58741500 & 1.60778100 \\ \mathrm{H} & 0.54262400 & 4.39743200 & 1.55288300 \\ \mathrm{H} & -0.47698700 & 2.22164500 & 0.80038300\end{array}$

\section{Cystal structure : $(E=-2001.42802248$ a.u $)$}

\begin{tabular}{|c|c|c|c|c|}
\hline Mo & -1 & 6.16300000 & 12.63400000 & 2.48900000 \\
\hline $\mathrm{Cl}$ & -1 & 5.41500000 & 10.87200000 & 1.11100000 \\
\hline $\mathrm{Cl}$ & -1 & 7.16200000 & 14.68600000 & 3.14300000 \\
\hline $\mathrm{N}$ & -1 & 4.64500000 & 13.16700000 & 3.13600000 \\
\hline $\mathrm{N}$ & -1 & 8.08900000 & 12.46300000 & 1.32700000 \\
\hline $\mathrm{N}$ & -1 & 5.98000000 & 13.74400000 & 0.50100000 \\
\hline $\mathrm{O}$ & -1 & 6.83100000 & 11.67100000 & 3.70000000 \\
\hline $\mathrm{C}$ & -1 & 9.10700000 & 11.76800000 & 1.80900000 \\
\hline $\mathrm{H}$ & 0 & 8.93931800 & 11.30841200 & 2.79201300 \\
\hline $\mathrm{C}$ & -1 & 10.27800000 & 11.62700000 & 1.12200000 \\
\hline $\mathrm{H}$ & 0 & 11.09249800 & 11.03563400 & 1.55916800 \\
\hline $\mathrm{C}$ & -1 & 10.40900000 & 12.22600000 & -0.09900000 \\
\hline $\mathrm{H}$ & 0 & 11.33673100 & 12.12916000 & -0.67840100 \\
\hline $\mathrm{C}$ & -1 & 9.36200000 & 12.95400000 & -0.58700000 \\
\hline $\mathrm{H}$ & 0 & 9.45000600 & 13.44439400 & -1.56320900 \\
\hline $\mathrm{C}$ & -1 & 8.20500000 & 13.06200000 & 0.14200000 \\
\hline $\mathrm{C}$ & -1 & 7.03000000 & 13.79400000 & -0.31700000 \\
\hline $\mathrm{C}$ & -1 & 6.97300000 & 14.47500000 & -1.50000000 \\
\hline $\mathrm{H}$ & 0 & 7.84981700 & 14.52242900 & -2.15598000 \\
\hline $\mathrm{C}$ & -1 & 5.81700000 & 15.09700000 & -1.87100000 \\
\hline $\mathrm{H}$ & 0 & 5.76113400 & 15.64943900 & -2.81815300 \\
\hline $\mathrm{C}$ & -1 & 4.73300000 & 15.00000000 & -1.04900000 \\
\hline $\mathrm{H}$ & 0 & 3.77484500 & 15.46540800 & -1.31346300 \\
\hline $\mathrm{C}$ & -1 & 4.84600000 & 14.33000000 & 0.11900000 \\
\hline $\mathrm{H}$ & 0 & 4.00192700 & 14.23567000 & 0.81342600 \\
\hline $\mathrm{C}$ & -1 & 3.61200000 & 13.88100000 & 3.66600000 \\
\hline $\mathrm{C}$ & -1 & 3.60300000 & 15.24700000 & 3.39200000 \\
\hline $\mathrm{H}$ & 0 & 4.41474400 & 15.65618600 & 2.77765500 \\
\hline $\mathrm{C}$ & -1 & 2.66000000 & 16.05400000 & 3.94600000 \\
\hline $\mathrm{H}$ & 0 & 2.67492300 & 17.13604800 & 3.75439200 \\
\hline $\mathrm{C}$ & -1 & 1.69700000 & 15.49700000 & 4.73600000 \\
\hline $\mathrm{H}$ & 0 & 0.91876800 & 16.13428700 & 5.18050400 \\
\hline $\mathrm{C}$ & -1 & 1.68500000 & 14.16400000 & 4.98000000 \\
\hline $\mathrm{H}$ & 0 & 0.88714700 & 13.76748600 & 5.61825100 \\
\hline $\mathrm{C}$ & -1 & 2.63400000 & 13.30500000 & 4.48100000 \\
\hline $\mathrm{C}$ & -1 & 2.61900000 & 11.82300000 & 4.77900000 \\
\hline $\mathrm{C}$ & -1 & 3.87100000 & 11.41300000 & 5.53400000 \\
\hline $\mathrm{H}$ & 0 & 4.79832200 & 11.59124700 & 4.96646900 \\
\hline $\mathrm{H}$ & 0 & 3.83006500 & 10.33200600 & 5.76840700 \\
\hline $\mathrm{H}$ & 0 & 3.95170800 & 11.96040800 & 6.49246100 \\
\hline $\mathrm{C}$ & -1 & 2.50700000 & 11.04200000 & 3.49100000 \\
\hline $\mathrm{H}$ & 0 & 1.57864100 & 11.30369300 & 2.94669600 \\
\hline $\mathrm{H}$ & 0 & 2.47151200 & 9.95600000 & 3.70351600 \\
\hline $\mathrm{H}$ & 0 & 3.35548300 & 11.20915200 & 2.80914800 \\
\hline $\mathrm{C}$ & -1 & 1.43000000 & 11.44900000 & 5.64900000 \\
\hline $\mathrm{H}$ & 0 & 1.45483200 & 11.94854000 & 6.63619100 \\
\hline $\mathrm{H}$ & 0 & 1.44314900 & 10.35937200 & 5.83737500 \\
\hline 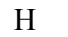 & 0 & 0.46153700 & 11.68310200 & 5.16654100 \\
\hline
\end{tabular}


(a) Complex with $\mathrm{CH}_{2} \underline{\mathrm{Cl}_{2}}(\mathrm{E}=-2960.72551550$ a.u $)$

\begin{tabular}{|c|c|c|c|c|}
\hline Mo & -1 & 14.52255500 & 14.31594000 & 9.93404800 \\
\hline $\mathrm{Cl}$ & -1 & 14.78802400 & 16.46893800 & 9.00970600 \\
\hline 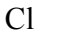 & -1 & 14.11819300 & 12.60700000 & 11.53236800 \\
\hline $\mathrm{N}$ & -1 & 13.17613900 & 13.75578300 & 8.99466800 \\
\hline$N$ & -1 & 15.84493200 & 15.07943600 & 11.59330800 \\
\hline$N$ & -1 & 13.28834100 & 15.53317100 & 11.42041100 \\
\hline $\mathrm{O}$ & -1 & 15.87285700 & 13.61736900 & 9.20584600 \\
\hline $\mathrm{C}$ & -1 & 17.14381700 & 14.82510800 & 11.59109200 \\
\hline $\mathrm{H}$ & 0 & 17.50442800 & 14.22069400 & 10.74828700 \\
\hline $\mathrm{C}$ & -1 & 17.97504800 & 15.29028000 & 12.56950600 \\
\hline $\mathrm{H}$ & 0 & 19.04795700 & 15.06821500 & 12.51736200 \\
\hline $\mathrm{C}$ & -1 & 17.45209300 & 16.03741500 & 13.58670800 \\
\hline $\mathrm{H}$ & 0 & 18.09531800 & 16.43393900 & 14.38301000 \\
\hline $\mathrm{C}$ & -1 & 16.10908000 & 16.28531000 & 385200 \\
\hline $\mathrm{H}$ & 0 & 15.67170100 & 16.88072000 & 14.40326800 \\
\hline $\mathrm{C}$ & -1 & 15.31776900 & 15.79782100 & 12.58550500 \\
\hline $\mathrm{C}$ & -1 & 13.88274000 & 16.04046000 & 12.49956000 \\
\hline $\mathrm{C}$ & -1 & 13.17465000 & 16.74735100 & 13.43084900 \\
\hline $\mathrm{H}$ & 0 & 13.67256300 & 17.14487000 & 14.32253200 \\
\hline $\mathrm{C}$ & -1 & 11.83942000 & 16.96284900 & 13.24813000 \\
\hline $\mathrm{H}$ & 0 & 11.26020500 & 17.52629400 & 13.99143600 \\
\hline $\mathrm{C}$ & -1 & 11.25055000 & 16.48068000 & 12.11741000 \\
\hline $\mathrm{H}$ & 0 & 10.18455100 & 16.64631100 & 11.91550700 \\
\hline $\mathrm{C}$ & -1 & 11.99144000 & 15.77276000 & 11.23578900 \\
\hline $\mathrm{H}$ & 0 & 11.55424100 & 15.35888400 & 10.31861400 \\
\hline $\mathrm{C}$ & -1 & 12.05817000 & 13.13033600 & 8.53042800 \\
\hline $\mathrm{C}$ & -1 & 11.13878100 & 12.71702000 & 9.49265500 \\
\hline $\mathrm{H}$ & 0 & 11.36882400 & 12.91714000 & 10.54663100 \\
\hline $\mathrm{C}$ & -1 & 10.03503200 & 12.01167000 & 9.13239600 \\
\hline $\mathrm{H}$ & 0 & 9.32896800 & 11.65889000 & 9.89701900 \\
\hline $\mathrm{C}$ & -1 & 9.81854800 & 11.75559300 & 7.80971000 \\
\hline $\mathrm{H}$ & 0 & 8.92261200 & 11.19797400 & 7.50050700 \\
\hline $\mathrm{C}$ & -1 & 10.69513000 & 12.18191000 & 6.86750900 \\
\hline $\mathrm{H}$ & 0 & 10.47199100 & 11.95294400 & 5.81930600 \\
\hline $\mathrm{C}$ & -1 & 11.84784200 & 12.86430700 & 7.17497100 \\
\hline $\mathrm{C}$ & -1 & 12.82576000 & 13.31559000 & 6.11469000 \\
\hline $\mathrm{C}$ & -1 & 14.17696000 & 12.65064000 & 6.31081000 \\
\hline $\mathrm{H}$ & 0 & 14.64817100 & 12.90037300 & 7.27482200 \\
\hline $\mathrm{H}$ & 0 & 14.87129900 & 12.97061200 & 5.51049000 \\
\hline $\mathrm{H}$ & 0 & 14.08704700 & 11.54910700 & 6.25500100 \\
\hline $\mathrm{C}$ & -1 & 12.97287000 & 14.81769000 & 6.15809000 \\
\hline $\mathrm{H}$ & 0 & 12.00153300 & 15.31809900 & 5.97772300 \\
\hline $\mathrm{H}$ & 0 & 13.67172900 & 15.15702900 & 5.36934900 \\
\hline $\mathrm{H}$ & 0 & 13.36719600 & 15.18486300 & 7.11836500 \\
\hline $\mathrm{C}$ & -1 & 12.34175000 & 12.94005000 & 4.72353000 \\
\hline $\mathrm{H}$ & 0 & 12.24596800 & 11.84566800 & 4.59077600 \\
\hline $\mathrm{H}$ & 0 & 13.07350200 & 13.29416500 & 3.97390100 \\
\hline $\mathrm{H}$ & 0 & 11.36945100 & 13.40613500 & 4.47300200 \\
\hline $\mathrm{C}$ & -1 & 18.29591100 & 17.84909900 & 9.16128200 \\
\hline $\mathrm{H}$ & 0 & 17.23885900 & 18.14439000 & 9.26306500 \\
\hline $\mathrm{H}$ & 0 & 18.37331200 & 16.76031400 & 9.00515900 \\
\hline $\mathrm{Cl}$ & -1 & 19.04825500 & 18.69485900 & 7.87215700 \\
\hline $\mathrm{Cl}$ & -1 & 19.15369500 & 18.26509500 & 10.65765100 \\
\hline
\end{tabular}


(b) Complex with $\mathrm{CH}_{2} \underline{\mathbf{C l}}_{2}(\mathrm{E}=-2960.72258477$ a.u $)$

\begin{tabular}{|c|c|c|c|c|}
\hline Mo & -1 & 7.10755000 & 11.35259000 & 12.23741000 \\
\hline $\mathrm{Cl}$ & -1 & 8.49859000 & 13.25635000 & 12.26704000 \\
\hline $\mathrm{Cl}$ & -1 & 5.19981000 & 9.97645000 & 12.56175000 \\
\hline $\mathrm{N}$ & -1 & 8.04559300 & 10.28677600 & 13.23395500 \\
\hline $\mathrm{N}$ & -1 & 5.53320000 & 12.75733000 & 11.44198000 \\
\hline $\mathrm{N}$ & -1 & 6.14950000 & 12.50864000 & 13.95794000 \\
\hline $\mathrm{O}$ & -1 & 7.45293000 & 10.87642000 & 10.65775000 \\
\hline $\mathrm{C}$ & -1 & 5.30034000 & 12.84193000 & 10.14179000 \\
\hline $\mathrm{H}$ & 0 & 5.91237600 & 12.18440600 & 9.51036200 \\
\hline $\mathrm{C}$ & -1 & $\begin{array}{ll}4.36900000 & 1\end{array}$ & 13.69732000 & 9.62655000 \\
\hline $\mathrm{H}$ & 0 & $\begin{array}{lll}4.21524000 & 1\end{array}$ & 13.73738800 & 8.54074100 \\
\hline $\mathrm{C}$ & -1 & 3.64944000 & 14.48799000 & 10.47718000 \\
\hline $\mathrm{H}$ & 0 & 2.89581500 & 15.18897000 & 10.09453800 \\
\hline $\mathrm{C}$ & -1 & 3.88173000 & 14.38722000 & 11.81916000 \\
\hline $\mathrm{H}$ & 0 & 3.31107000 & 15.01063700 & 12.51692600 \\
\hline $\mathrm{C}$ & -1 & 4.83093000 & 13.51441000 & 12.28586000 \\
\hline $\mathrm{C}$ & -1 & 5.16325000 & 13.36581900 & 13.69762000 \\
\hline $\mathrm{C}$ & -1 & 4.54061000 & 14.05386000 & 14.70119000 \\
\hline $\mathrm{H}$ & 0 & 3.71941100 & 14.74368100 & 14.47540700 \\
\hline $\mathrm{C}$ & -1 & 4.94567000 & 13.88408000 & 15.99335000 \\
\hline $\mathrm{H}$ & 0 & 4.45031100 & 14.42897300 & 16.80771100 \\
\hline $\mathrm{C}$ & -1 & 5.98709000 & 13.04109000 & 16.24405000 \\
\hline $\mathrm{H}$ & 0 & 6.36269600 & 12.88770000 & 17.26382200 \\
\hline $\mathrm{C}$ & -1 & 6.55759000 & 12.37082000 & 15.21816000 \\
\hline $\mathrm{H}$ & 0 & 7.39141600 & 11.67575400 & 15.37719700 \\
\hline $\mathrm{C}$ & -1 & 8.48148500 & $9.31727700 \quad 1$ & 14.08657600 \\
\hline $\mathrm{C}$ & -1 & 7.57625800 & $8.90085000 \quad 1$ & 15.06081000 \\
\hline $\mathrm{H}$ & 0 & 6.58523800 & 9.37130000 & 15.07930700 \\
\hline $\mathrm{C}$ & -1 & 7.89053800 & 7.88159200 & 15.90209700 \\
\hline $\mathrm{H}$ & 0 & 7.16174800 & $\begin{array}{ll}7.53361500 & 1\end{array}$ & 16.64739700 \\
\hline $\mathrm{C}$ & -1 & 9.12353200 & $\begin{array}{ll}7.30499100 & 1\end{array}$ & 15.80797500 \\
\hline $\mathrm{H}$ & 0 & 9.40010200 & $6.48374000 \quad 1$ & 16.48480400 \\
\hline $\mathrm{C}$ & -1 & 10.01817100 & 7.72620100 & 14.88029500 \\
\hline $\mathrm{H}$ & 0 & 10.99605400 & 7.23215800 & 14.85903300 \\
\hline $\mathrm{C}$ & -1 & 9.74291700 & $8.72604900 \quad 1$ & 13.97846100 \\
\hline $\mathrm{C}$ & -1 & 10.74376400 & 9.17182800 & 12.93678000 \\
\hline $\mathrm{C}$ & -1 & 10.21084100 & 8.92381100 & 11.53693500 \\
\hline $\mathrm{H}$ & 0 & 9.28884600 & 9.48531800 & 11.31701000 \\
\hline $\mathrm{H}$ & 0 & 10.96805700 & 9.22509400 & 10.78800900 \\
\hline $\mathrm{H}$ & 0 & 9.99475900 & 7.84916300 & 11.38084900 \\
\hline $\mathrm{C}$ & -1 & 11.05891900 & 10.63657300 & 13.12352100 \\
\hline $\mathrm{H}$ & 0 & 11.48233300 & 10.82667000 & 14.12894800 \\
\hline $\mathrm{H}$ & 0 & 11.81043200 & 10.96535200 & 12.37984300 \\
\hline $\mathrm{H}$ & 0 & 10.17830900 & $\begin{array}{ll}0 & 11.28608300\end{array}$ & O 13.00041000 \\
\hline $\mathrm{C}$ & -1 & 12.04757300 & 8.40028700 & 13.06157200 \\
\hline $\mathrm{H}$ & 0 & 11.91616800 & 7.31404800 & 12.89445400 \\
\hline $\mathrm{H}$ & 0 & 12.76092900 & 8.75939000 & 12.29681800 \\
\hline $\mathrm{H}$ & 0 & 12.53028800 & 8.53550400 & 14.04775000 \\
\hline $\mathrm{C}$ & -1 & 11.35199500 & 4.18147500 & 13.78575000 \\
\hline $\mathrm{H}$ & 0 & 11.01599400 & 5.04665200 & 13.18852500 \\
\hline $\mathrm{H}$ & 0 & 11.89943800 & 3.45262200 & 13.16665700 \\
\hline $\mathrm{Cl}$ & -1 & 10.01022900 & 3.43602700 & 14.55150200 \\
\hline $\mathrm{Cl}$ & -1 & 12.44255500 & 4.77323400 & 15.05314100 \\
\hline
\end{tabular}


(c) Complex with 2-CH${ }_{2} \underline{\mathbf{C l}}_{2}:(\mathrm{E}=-3920.02123874 \mathrm{a} . \mathrm{u})$

\begin{tabular}{|c|c|c|c|c|}
\hline Mo & -1 & 14.52269000 & ) 14.31587100 & $\begin{array}{l}0 \quad 9.93396100\end{array}$ \\
\hline & -1 & 14.78736700 & 16.46880000 & 9.00974600 \\
\hline & -1 & 14.11814900 & 12.60700100 & 11.53235900 \\
\hline & -1 & 13.17615900 & 13.75576500 & 8.99465300 \\
\hline & -1 & 15.84491700 & 15.07949300 & 11.59335700 \\
\hline & -1 & 13.28832500 & 15.53316000 & 11.42041800 \\
\hline & -1 & 15.87288700 & 13.61738300 & 9.20588800 \\
\hline & -1 & 17.14360100 & 14.82488800 & 11.59118900 \\
\hline & 0 & 17.50399900 & 14.22041500 & 10.74834700 \\
\hline & -1 & 17.97511100 & 15.29024400 & 12.56948900 \\
\hline & 0 & 19.04802700 & 15.06796000 & 12.51758200 \\
\hline & -1 & 17.45209200 & 16.03747900 & 13.58666500 \\
\hline & 0 & 18.09535400 & 16.43391600 & 14.38298900 \\
\hline & -1 & 16.10908000 & 16.28530900 & 13.59384700 \\
\hline & 0 & 15.67159900 & 16.88069900 & 14.40319500 \\
\hline & -1 & 15.31777400 & 15.79785300 & 12.58548600 \\
\hline & -1 & 13.88273700 & 16.04046100 & 12.49955600 \\
\hline & -1 & 13.17465700 & 16.74737300 & 86500 \\
\hline & 0 & 13.67236900 & 17.14602800 & 14.32203700 \\
\hline & -1 & 40500 & 16.96 & 13.2 \\
\hline & 0 & 11.2 & 17.52 & 13.9 \\
\hline & -1 & 11.25078500 & 16.48061600 & 12.11748900 \\
\hline & 0 & 10.18692800 & 16.65544800 & 11.91112300 \\
\hline & -1 & 11.9 & 15.77 & 11.2 \\
\hline & 0 & 11.55528600 & 15.37056800 & 10.31297900 \\
\hline & -1 & 12.05817100 & 13.13034600 & 43300 \\
\hline & -1 & 11.13877800 & 12.71702000 & 9.49 \\
\hline & 0 & 11.37006900 & 12.91671700 & 10.54633400 \\
\hline & -1 & 10.03502700 & 12.01167000 & 9.13240700 \\
\hline & 0 & 9.33231400 & 11.65285000 & 2500 \\
\hline & -1 & 9.81855300 & 11.75558500 & 7.80971000 \\
\hline & 0 & 8.92527100 & 11.19347600 & 7.50046800 \\
\hline & -1 & 10.69513100 & 12.18191000 & 51100 \\
\hline & 0 & 10.4 & & \\
\hline & -1 & 11.84783700 & 12.86431500 & 7.17496900 \\
\hline & -1 & 12.82576000 & 13.31559100 & 6.11469000 \\
\hline & -1 & 14.17696000 & 12.65064000 & 81000 \\
\hline & 0 & 14.64808300 & 12.89952700 & 7.27511100 \\
\hline & 0 & 14.87133000 & 12.97189200 & 5.51107400 \\
\hline & 0 & 14.08774400 & 11.54906000 & 6.25364200 \\
\hline & -1 & 12.97287000 & 14.81769000 & 6.15809000 \\
\hline & 0 & 12.00080300 & 15.31822100 & 5.98214100 \\
\hline 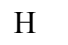 & 0 & 13.66904600 & 15.15665000 & 5.36708200 \\
\hline & 0 & 13.37047400 & 15.18473700 & 7.11707800 \\
\hline & -1 & 12.34175000 & 12.94004900 & 4.72353000 \\
\hline $\mathrm{H}$ & 0 & 12.24554400 & 11.84567600 & 4.59043200 \\
\hline & 0 & 13.07368500 & 13.29379500 & 3.97400900 \\
\hline & 0 & 11.36990900 & 13.40698400 & 4.47279100 \\
\hline & -1 & 18.48596400 & 17.89437400 & 9.17536300 \\
\hline $\mathrm{H}$ & 0 & 17.42881500 & 18.19083500 & 9.27675900 \\
\hline & 0 & 18.56512800 & 16.80574300 & 9.01864300 \\
\hline & -1 & 19.23700200 & 18.73967100 & 7.88595900 \\
\hline $\mathrm{Cl}$ & -1 & 19.34263100 & 18.30991800 & 10.67169200 \\
\hline & -1 & 8.20923500 & 15.56456400 & 9.05441400 \\
\hline & 0 & 8.32562500 & 15.61542400 & 10.15006200 \\
\hline & 0 & 8.08781200 & 14.52044000 & 8.72033700 \\
\hline & -1 & 9.55890400 & 16.27729000 & 8.27150500 \\
\hline
\end{tabular}


(d) Complex with 2-CH $2 \underline{C l}_{2}$ : $(\mathrm{E}=-3920.01991014$ a.u $)$

\begin{tabular}{|c|c|c|c|c|}
\hline \multicolumn{5}{|l|}{01} \\
\hline Mo & -1 & 7.10762600 & 11.35255000 & 12.2 \\
\hline $\mathrm{Cl}$ & -1 & 8.49852200 & 13.25638700 & 12.26687400 \\
\hline $\mathrm{Cl}$ & -1 & 5.19973700 & 9.97650700 & 12.56155400 \\
\hline $\mathrm{N}$ & -1 & 8.04565800 & 10.28674100 & 5900 \\
\hline $\mathrm{N}$ & -1 & 5.53317200 & 12.75732600 & 11.44198800 \\
\hline $\mathrm{N}$ & -1 & 6.14953000 & 12.50863000 & 13.95796400 \\
\hline $\mathrm{O}$ & -1 & 7.45291900 & 10.87642800 & 10.65774500 \\
\hline $\mathrm{C}$ & -1 & 5.30039600 & 12.84197300 & 180900 \\
\hline $\mathrm{H}$ & 0 & 5.90967000 & 12.18232400 & 9.50989100 \\
\hline $\mathrm{C}$ & -1 & 4.36898800 & 13.69724600 & 9.62654400 \\
\hline $\mathrm{H}$ & 0 & 4.22193600 & 13.74517500 & 8.54061200 \\
\hline $\mathrm{C}$ & -1 & 3.64940000 & 14.48796400 & 10.47714900 \\
\hline $\mathrm{H}$ & 0 & 2.90018800 & 15.19261600 & 10.09349500 \\
\hline $\mathrm{C}$ & -1 & 3.88173300 & 14.38724400 & 11.81916000 \\
\hline $\mathrm{H}$ & 0 & 3.31218900 & 15.01187500 & 12.51659100 \\
\hline $\mathrm{C}$ & -1 & 4.83093200 & 13.51441400 & 12.28586100 \\
\hline $\mathrm{C}$ & -1 & 5.16327700 & 13.36584800 & 13.69761700 \\
\hline $\mathrm{C}$ & -1 & 4.54060800 & 14.05386200 & 14.70119100 \\
\hline $\mathrm{H}$ & 0 & 3.71932000 & 14.74332800 & 14.47502000 \\
\hline $\mathrm{C}$ & -1 & 4.94566900 & 13.88 & 15.9 \\
\hline $\mathrm{H}$ & 0 & 4.45021900 & 14.42888400 & 16.80768300 \\
\hline $\mathrm{C}$ & -1 & 5.98708600 & 13.04109100 & 4400 \\
\hline $\mathrm{H}$ & 0 & 4100 & 4300 & 0800 \\
\hline $\mathrm{C}$ & -1 & 6.55758900 & 12.37081200 & 15.21816400 \\
\hline $\mathrm{H}$ & 0 & 7.39116300 & 11.67555000 & 15.37783400 \\
\hline $\mathrm{C}$ & -1 & 8.48149500 & 9.31729300 & 14.0 \\
\hline $\mathrm{C}$ & -1 & 7.57625300 & 8.900 & 15.06083900 \\
\hline $\mathrm{H}$ & 0 & 6.58533000 & 9.37144800 & 15.0 \\
\hline $\mathrm{C}$ & -1 & 7.89058300 & 7.88164600 & 15.90210200 \\
\hline $\mathrm{H}$ & 0 & 7.16164900 & 7.533 & 07900 \\
\hline $\mathrm{C}$ & -1 & 9.123 & 7.304 & 74000 \\
\hline $\mathrm{H}$ & 0 & 9.40001900 & 6.48353900 & 16.48457300 \\
\hline $\mathrm{C}$ & -1 & 10.01838000 & 7.72693400 & 14.88110400 \\
\hline $\mathrm{H}$ & 0 & 10.99652700 & 7.23343900 & 14.86025200 \\
\hline $\mathrm{C}$ & -1 & 9.74263600 & 8.72555900 & 13.97799600 \\
\hline $\mathrm{C}$ & -1 & 10.74371100 & 9.17185700 & 12.93724900 \\
\hline $\mathrm{C}$ & -1 & 10.21090900 & 8.92386900 & 11.53689900 \\
\hline $\mathrm{H}$ & 0 & 9.28828500 & 9.48434400 & 11.31714900 \\
\hline $\mathrm{H}$ & 0 & 10.96812900 & 9.22578700 & 10.78832500 \\
\hline $\mathrm{H}$ & 0 & 9.99576700 & 7.84919400 & 11.38048500 \\
\hline $\mathrm{C}$ & -1 & 11.05895300 & 10.63658800 & 13.12335000 \\
\hline $\mathrm{H}$ & 0 & 11.48348900 & 10.82712300 & 14.12814100 \\
\hline $\mathrm{H}$ & 0 & 11.80958700 & 10.96519200 & 12.37881800 \\
\hline $\mathrm{H}$ & 0 & 10.17799400 & 11.28555400 & 13.00142100 \\
\hline $\mathrm{C}$ & -1 & 12.04769900 & 8.40039700 & 13.06135000 \\
\hline $\mathrm{H}$ & 0 & 11.91605000 & 7.31433000 & 12.89396700 \\
\hline $\mathrm{H}$ & 0 & 12.76074600 & 8.75972200 & 12.29650600 \\
\hline $\mathrm{H}$ & 0 & 12.53070600 & 8.53546800 & 14.04736000 \\
\hline $\mathrm{C}$ & -1 & 8.23642100 & 15.47146500 & 9.22363600 \\
\hline $\mathrm{H}$ & 0 & 8.35234300 & 15.50757700 & 10.31916500 \\
\hline $\mathrm{H}$ & 0 & 8.10318000 & 14.42909400 & 8.88963300 \\
\hline $\mathrm{Cl}$ & -1 & 9.58589300 & 16.18457400 & 8.44050700 \\
\hline $\mathrm{Cl}$ & -1 & 6.79260200 & 16.39655700 & 8.76972000 \\
\hline $\mathrm{C}$ & -1 & 11.35222100 & 4.18087700 & 13.78544800 \\
\hline
\end{tabular}




$\begin{array}{lrrrrr}\mathrm{H} & 0 & 11.01658500 & 5.04562100 & 13.18750700 \\ \mathrm{H} & 0 & 11.89984100 & 3.45139600 & 13.16731300 \\ \mathrm{Cl} & -1 & 10.01017600 & 3.43623500 & 14.55161700 \\ \mathrm{Cl} & -1 & 12.44236700 & 4.77336500 & 15.05312200\end{array}$

Supplementary Date Table S7: Comparison of structural and QTAIM parameters of Energy minimised (Emin), X-ray (xray), and the complexes (a-d), structures. 


\begin{tabular}{|c|c|c|c|c|c|c|c|c|}
\hline Parameter & Distance $^{a}$ & $\mathbf{X} \cdots \cdot \mathbf{H}-\mathrm{C}^{\mathrm{b}}$ & bcp & $\rho^{f}$ & $|\mathbf{V}| / \mathbf{G}$ & $\mathbf{E}^{\mathrm{g}}$ & $q(\mathrm{~A})^{\mathrm{h}}$ & $q(\mathrm{~B})^{\mathrm{h}}$ \\
\hline \multicolumn{9}{|c|}{$M o=O(1) \cdots \cdot H(18 a)$} \\
\hline Emin $^{\mathrm{d}}$ & 2.586 & 172.2 & no & - & - & - & -0.7796 & 0.0308 \\
\hline X-ray ${ }^{\mathrm{c}}$ & 2.53 & 166.8 & yes & 0.0110 & 0.9348 & 2.41 & -0.7887 & 0.0342 \\
\hline (a) & 2.397 & 172.5 & yes & 0.0111 & 0.9350 & 2.41 & -0.7910 & 0.0328 \\
\hline (b) & 2.396 & 172.8 & yes & 0.0110 & 0.9348 & 2.41 & -0.7901 & 0.0362 \\
\hline (c) & 2.397 & 172.5 & yes & 0.0110 & 0.9347 & 2.41 & -0.7897 & 0.0328 \\
\hline (d) & 2.396 & 172.7 & yes & 0.0110 & 0.9349 & 2.41 & -0.7924 & 0.0348 \\
\hline \multicolumn{9}{|c|}{$M o=O(1) \cdots H(1)$} \\
\hline Emin $^{\mathrm{d}}$ & 2.380 & 116.6 & yes & 0.0131 & 0.9126 & 2.91 & -0.7796 & 0.1100 \\
\hline$X-$ ray $^{\mathrm{c}}$ & 2.44 & 114.0 & yes & 0.0148 & 0.9189 & 3.38 & -0.7887 & 0.1067 \\
\hline (a) & 2.324 & 115.0 & yes & 0.0148 & 0.9194 & 3.39 & -0.7910 & 0.1004 \\
\hline (b) & 2.325 & 115.0 & yes & 0.0148 & 0.9190 & 3.38 & -0.7901 & 0.1065 \\
\hline (c) & 2.324 & 115.0 & yes & 0.0148 & 0.9190 & 3.39 & -0.7897 & 0.1007 \\
\hline (d) & 2.325 & 115.0 & yes & 0.0148 & 0.9196 & 3.39 & -0.7924 & 0.1002 \\
\hline \multicolumn{9}{|c|}{$M_{0}=N(1) \cdots H(18 a)$} \\
\hline Emin $^{d}$ & 2.407 & 118.7 & yes & 0.0139 & 0.8778 & 2.65 & -1.0455 & 0.0308 \\
\hline X-ray ${ }^{c}$ & 2.41 & 124.2 & yes & 0.0138 & 0.8705 & 2.67 & -1.0531 & 0.0342 \\
\hline (a) & 2.420 & 116.2 & yes & 0.0138 & 0.8701 & 2.67 & -1.0528 & 0.0328 \\
\hline (b) & 2.421 & 116.1 & yes & 0.0138 & 0.8707 & 2.66 & -1.0541 & 0.0362 \\
\hline (c) & 2.420 & 116.2 & yes & 0.0138 & 0.8706 & 2.67 & -1.0511 & 0.0328 \\
\hline (d) & 2.421 & 116.1 & yes & 0.0138 & 0.8700 & 2.66 & -1.0530 & 0.0348 \\
\hline \multicolumn{9}{|c|}{$\mathrm{Mo}=\mathbf{N}(1) \cdots \cdot \mathbf{H}(19 \mathrm{c})$} \\
\hline Emin $^{\mathrm{d}}$ & 2.466 & 118.4 & yes & 0.0118 & 0.8564 & 2.22 & -1.0455 & 0.0219 \\
\hline X-ray ${ }^{c}$ & 2.39 & 123.2 & yes & 0.0145 & 0.8690 & 2.82 & -1.0531 & 0.0215 \\
\hline (a) & 2.367 & 117.5 & yes & 0.0145 & 0.8693 & 2.82 & -1.0528 & 0.0186 \\
\hline (b) & 2.366 & 117.5 & yes & 0.0145 & 0.8695 & 2.82 & -1.0541 & 0.0222 \\
\hline (c) & 2.368 & 117.4 & yes & 0.0145 & 0.8692 & 2.82 & -1.0511 & 0.0188 \\
\hline (d) & 2.366 & 117.5 & yes & 0.0145 & 0.8697 & 2.82 & -1.0530 & 0.0193 \\
\hline \multicolumn{9}{|c|}{ Mo-Cl(1) $\cdots \cdot H(19 c)$} \\
\hline Emin $^{\mathrm{d}}$ & 2.722 & 163.8 & yes & 0.0095 & 0.8464 & 1.61 & -0.5532 & 0.0219 \\
\hline X-ray ${ }^{\mathrm{c}}$ & 2.82 & 162.4 & yes & 0.0102 & 0.8548 & 1.77 & -0.5656 & 0.0215 \\
\hline (a) & 2.692 & 163.9 & yes & 0.0101 & 0.8513 & 1.76 & -0.5739 & 0.0186 \\
\hline (b) & 2.690 & 164.1 & yes & 0.0101 & 0.8549 & 1.76 & -0.5667 & 0.0222 \\
\hline (c) & 2.691 & 164.1 & yes & 0.0101 & 0.8520 & 1.76 & -0.5716 & 0.0188 \\
\hline (d) & 2.699 & 164.0 & yes & 0.0101 & 0.8517 & 1.76 & -0.5742 & 0.0193 \\
\hline \multicolumn{9}{|c|}{$\mathrm{Mo}-\mathrm{Cl}(2) \cdots \cdot \mathrm{H}(12)$} \\
\hline Emin $^{\mathrm{d}}$ & 2.993 & 127.2 & yes & 0.0064 & 0.7823 & 0.97 & -0.5614 & 0.0402 \\
\hline$X-$ ray $^{\mathrm{c}}$ & 3.06 & 118.3 & yes & 0.0072 & 0.7829 & 1.15 & -0.5719 & 0.0284 \\
\hline (a) & 2.937 & 120.0 & yes & 0.0072 & 0.7825 & 1.15 & -0.5680 & 0.0298 \\
\hline (b) & 2.936 & 120.0 & yes & 0.0072 & 0.7835 & 1.15 & -0.5721 & 0.0290 \\
\hline (c) & 2.936 & 120.0 & yes & 0.0072 & 0.7833 & 1.15 & -0.5716 & 0.0301 \\
\hline (d) & 2.937 & 120.0 & yes & 0.0072 & 0.7830 & 1.15 & -0.5681 & 0.0296 \\
\hline $\mathbf{H}(\mathbf{1 0}) \cdots \mathbf{C}(1$ & & & & & & & & \\
\hline Emin $^{\mathrm{d}}$ & 2.888 & 135.8 & no & - & - & - & 0.0838 & 0.4192 \\
\hline$X-$ ray $^{\mathrm{c}}$ & 3.04 & 136.1 & no & - & - & - & 0.0771 & 0.4270 \\
\hline (a) & 2.902 & 137.4 & no & - & - & - & 0.0762 & 0.4273 \\
\hline (b) & 2.901 & 137.4 & no & - & - & - & 0.0772 & 0.4270 \\
\hline (c) & 2.908 & 136.9 & no & - & - & - & 0.0807 & 0.4319 \\
\hline (d) & 2.902 & 137.4 & no & - & - & - & 0.0764 & 0.4264 \\
\hline $\mathbf{H}(\mathbf{1 0}) \cdots \mathbf{C}(1$ & & & & & & & & \\
\hline Emin $^{\mathrm{d}}$ & 2.780 & 140.7 & yes & 0.0064 & 0.7361 & 0.98 & 0.0838 & -0.0238 \\
\hline$X-$ ray $^{c}$ & 2.91 & 132.2 & yes & 0.0064 & 0.7022 & 0.99 & 0.0771 & -0.0143 \\
\hline (a) & 2.790 & 131.5 & yes & 0.0064 & 0.7024 & 0.99 & 0.0762 & -0.0138 \\
\hline (b) & 2.799 & 131.5 & yes & 0.0064 & 0.7025 & 0.99 & 0.0772 & -0.0145 \\
\hline (c) & 2.809 & 130.6 & yes & 0.0063 & 0.7012 & 0.97 & 0.0807 & -0.0140 \\
\hline (d) & 2.799 & 131.5 & yes & 0.0064 & 0.7030 & 0.99 & 0.0764 & -0.0139 \\
\hline $\mathbf{H}(\mathbf{1 0}) \cdots \cdot \mathbf{N}(1$ & & & & & & & & \\
\hline Emin $^{\mathrm{d}}$ & 2.692 & 115.4 & no & - & - & - & 0.0838 & -1.0455 \\
\hline X-ray ${ }^{c}$ & 2.74 & 113.4 & no & - & - & - & 0.0771 & -1.0531 \\
\hline (a) & 2.637 & 113.8 & no & - & - & - & 0.0762 & -1.0528 \\
\hline (b) & 2.636 & 113.9 & no & - & - & - & 0.0772 & -1.0541 \\
\hline (c) & 2.641 & 113.6 & no & - & - & - & 0.0807 & -1.0531 \\
\hline (d) & 2.637 & 113.8 & no & - & - & - & 0.0764 & -1.0530 \\
\hline $\mathbf{H}(4) \cdots \mathbf{H}(7)$ & & & & & & & & \\
\hline Emin $^{d}$ & 2.063 & 116.0 & yes & 0.0115 & 0.8040 & 2.20 & 0.0336 & 0.0334 \\
\hline X-ray ${ }^{\mathrm{c}}$ & 2.14 & 116.1 & yes & 0.0128 & 0.8040 & 2.51 & 0.0252 & 0.0260 \\
\hline (a) & 2.018 & 115.8 & yes & 0.0128 & 0.8268 & 2.51 & 0.0261 & 0.0279 \\
\hline (b) & 2.018 & 115.9 & yes & 0.0128 & 0.8272 & 2.51 & 0.0253 & 0.0262 \\
\hline (c) & 2.019 & 115.9 & yes & 0.0128 & 0.8268 & 2.50 & 0.0264 & 0.0281 \\
\hline (d) & 2.018 & 115.8 & yes & 0.0128 & 0.8268 & 2.50 & 0.0262 & 0.0279 \\
\hline $\mathbf{C}(20) \cdots \cdot \mathbf{H}(1$ & & & & & & & & \\
\hline Emin $^{\mathrm{d}}$ & 2.428 & 101.8 & no & - & - & - & 0.0443 & 0.0157 \\
\hline X-ray ${ }^{d}$ & 2.40 & 105.3 & no & - & - & - & 0.0459 & 0.0076 \\
\hline (a) & 2.381 & 101.2 & no & - & - & - & 0.0452 & 0.0088 \\
\hline (b) & 2.387 & 100.8 & no & - & - & - & 0.0483 & 0.0148 \\
\hline (c) & 2.381 & 101.2 & no & - & - & - & 0.0451 & 0.0108 \\
\hline (d) & 2.388 & 100.8 & no & - & - & - & 0.0482 & 0.0159 \\
\hline
\end{tabular}




\begin{tabular}{|c|c|c|c|c|c|c|c|c|}
\hline \multicolumn{9}{|c|}{ H(20a) $\cdots \cdot$ H(15) } \\
\hline $\operatorname{Emin}^{\mathrm{d}}$ & 2.190 & 113.5 & no & - & - & - & -0.0253 & 0.0157 \\
\hline X-ray ${ }^{\mathrm{c}}$ & 2.18 & 112.8 & no & - & - & - & -0.0261 & 0.0076 \\
\hline (a) & 2.160 & 112.8 & no & - & - & - & -0.0254 & 0.0088 \\
\hline (b) & 2.171 & 112.2 & no & - & - & - & -0.0376 & 0.0148 \\
\hline (c) & 2.160 & 112.9 & no & - & - & - & -0.0259 & 0.0108 \\
\hline (d) & 2.172 & 112.2 & no & - & - & - & -0.0368 & 0.0159 \\
\hline \multicolumn{9}{|c|}{$\mathbf{H}(20 \mathrm{c}) \cdots \cdot \mathbf{H}(15)$} \\
\hline $\operatorname{Emin}^{\mathrm{d}}$ & 2.231 & 111.5 & no & - & - & - & -0.0253 & 0.0157 \\
\hline $\mathrm{X}-$ ray $^{\mathrm{c}}$ & 2.18 & 111.6 & no & - & - & - & -0.0284 & 0.0076 \\
\hline (a) & 2.174 & 111.6 & no & - & - & - & -0.0278 & 0.0088 \\
\hline (b) & 2.171 & 111.6 & no & - & - & - & -0.0221 & 0.0148 \\
\hline (c) & 2.176 & 111.6 & no & - & - & - & -0.0268 & 0.0108 \\
\hline (d) & 2.170 & 111.6 & no & - & - & - & -0.0215 & 0.0159 \\
\hline
\end{tabular}

${ }^{\mathrm{a}}$ Separation distance, $\AA{ }^{\mathrm{b}} ;{ }^{\mathrm{b}}$ degrees $\left({ }^{\mathrm{o}}\right) ;{ }^{\mathrm{c}} \mathrm{X}$-ray structure; ${ }^{\mathrm{d}}$ Energy minimised structure; ${ }^{\mathrm{f}} \rho(\mathrm{bcp})=e / \mathrm{Bohr}^{3} ;{ }^{\mathrm{g}}$ Interaction energy, ${ }^{13} \mathrm{kcal}$ mol ${ }^{-}$ ${ }^{1} ;{ }^{\mathrm{h}}$ atomic basin charge $(\mathrm{A}---\mathrm{B}), q=e$. 
Supplementary Figures S1-S10: Comparison of energy minimised (EM) and Crystal structure (SP) iso-surfaces.

(EM and SP related by inversion. Atom colours: red, O; blue, N; smaller blue-green, C; larger blue-green, $\mathrm{Cl}$; white, $\mathrm{H}$ ).

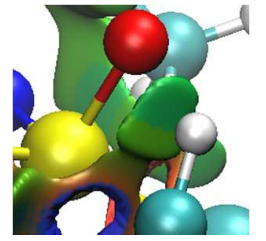

(EM)

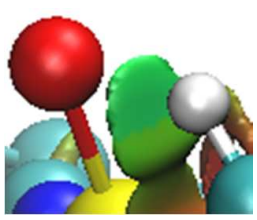

(CS)

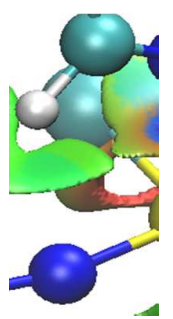

(EM)

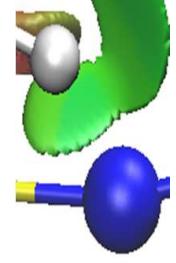

(CS)

Fig S2. $\mathrm{N}(1) \cdots \mathrm{H}(10)$ separation.

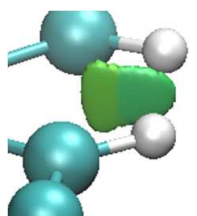

(EM)

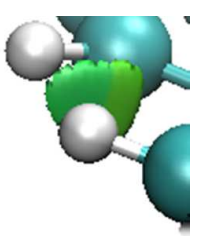

(CS)

Fig S3. $H(4) \cdots H(7)$ separation.

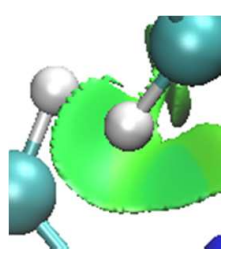

(EM)

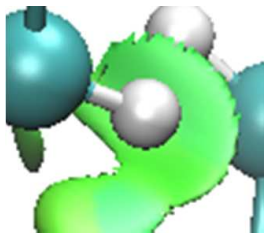

(CS)

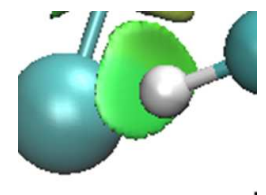

(EM)

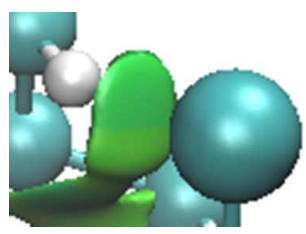

(CS)

Fig S4. $H(10) \cdots H(12)$ separation.

Fig S5. $\mathrm{Cl}(1) \cdots \mathrm{H}(19 \mathrm{c})$ separation

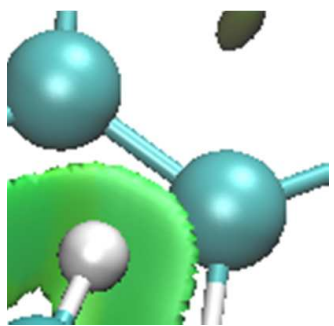

(EM)

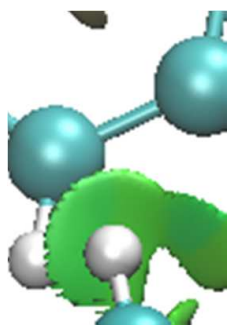

(CS) 
Fig S6. $C(12) \cdots H(10)$ separation.

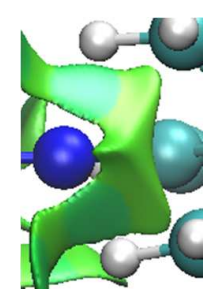

(EM)

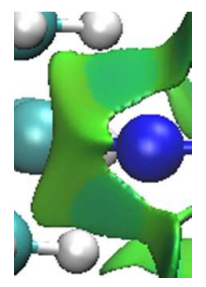

(CS)
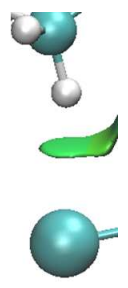

(EM)

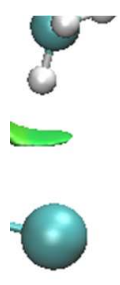

(CS)

Fig S7. $N(1) \cdots H(18 a)$ and $N(1) \cdots H(19 c)$ separations.

Fig S8. $\mathrm{Cl}(1) \cdots \mathrm{H}(19 \mathrm{c})$ separation.

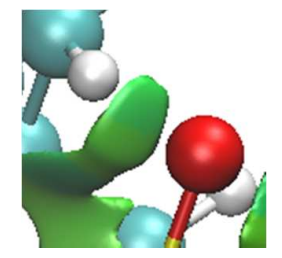

(EM)

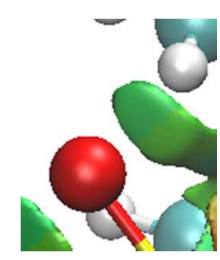

(CS)

Fig S9. $\mathrm{O}(1) \cdots \mathrm{H}(18 \mathrm{a})$ separation

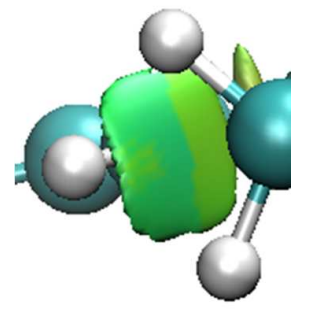

(EM)

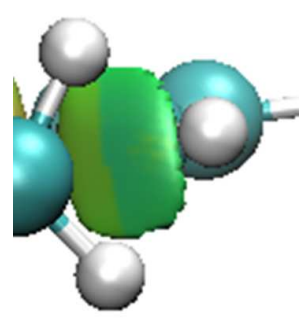

(CS)

Fig S10. $H(15) \cdots H(20 a), H(15) \cdots H(20 c)$ and $H(15) \cdots C(20)$ separations. 
Supplementary Figure S11: Molecular graphs for the calculated X-ray (X-rayC) and PBE-D3 energy minimised (Emin) structures.

\section{A) Molecular graph for the calculated X-ray structure}

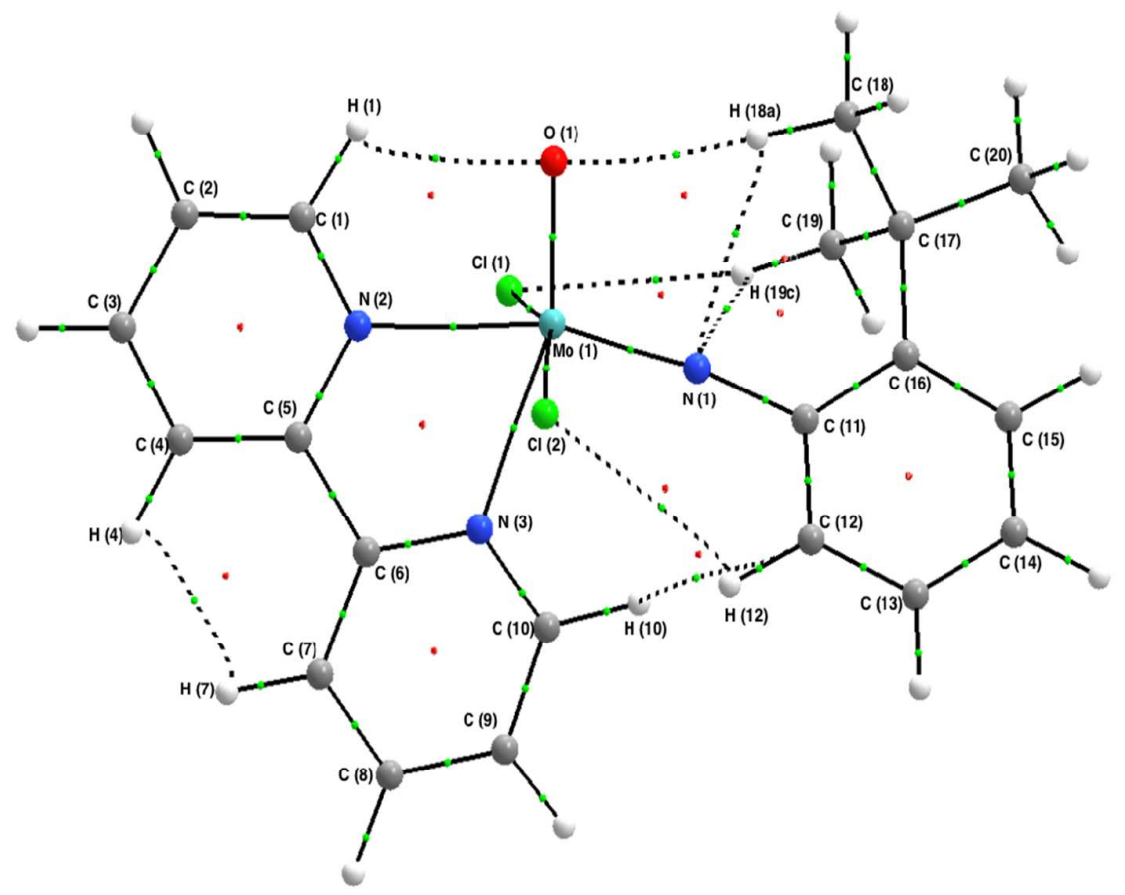

B) Molecular graph for the PBE-D3 energy minimised structure

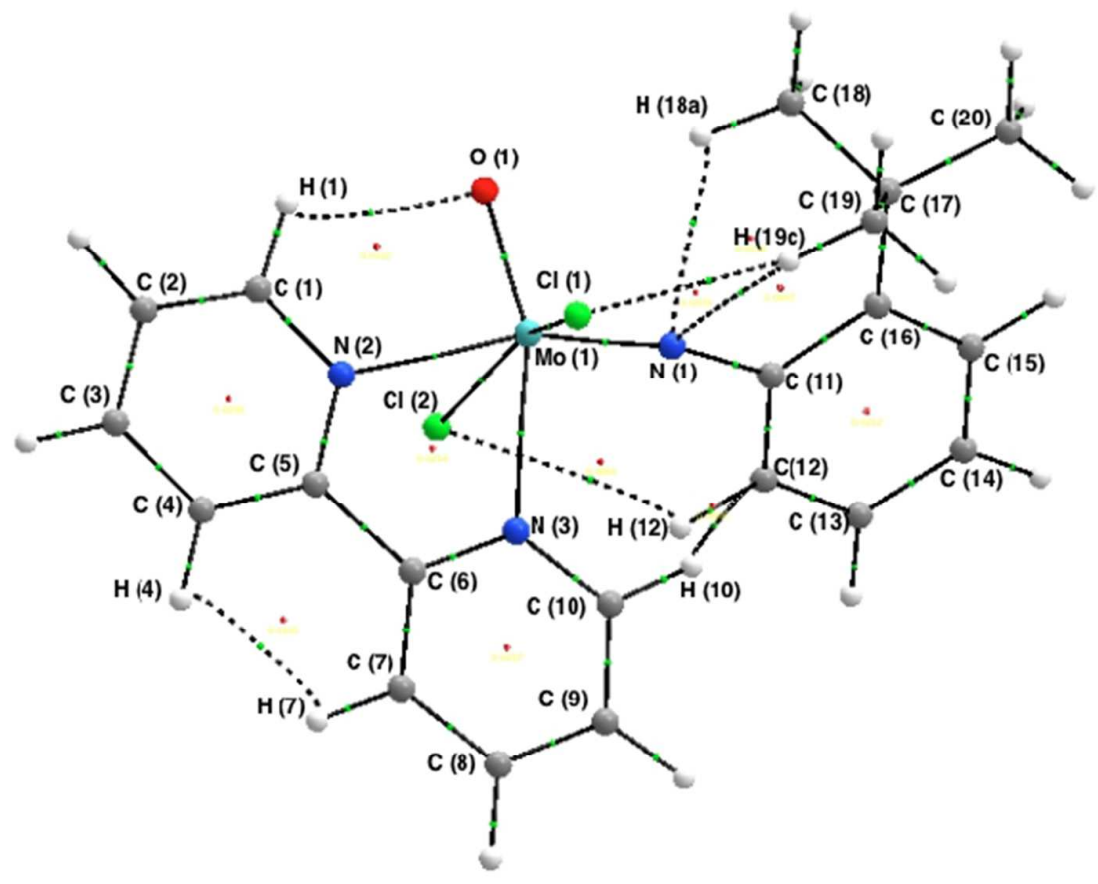


Supplementary Figure S12: Unit cell obtained from the X-ray crystal structure.

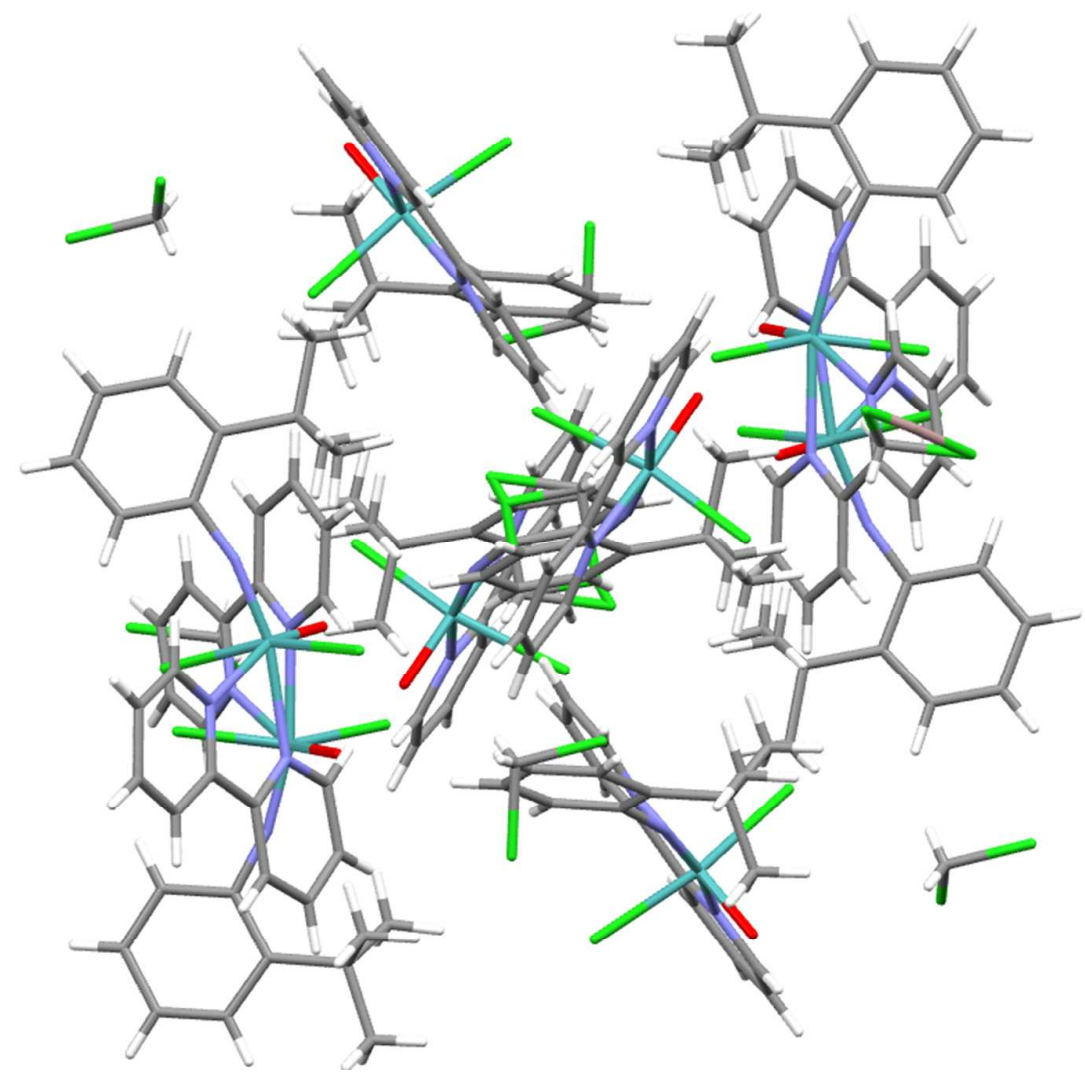

Supplementary Figure S13: Molecular graphs showing intermolecular bond critical points with dicholoromethane molecules for complexes a-d.

\section{Complex (a)}

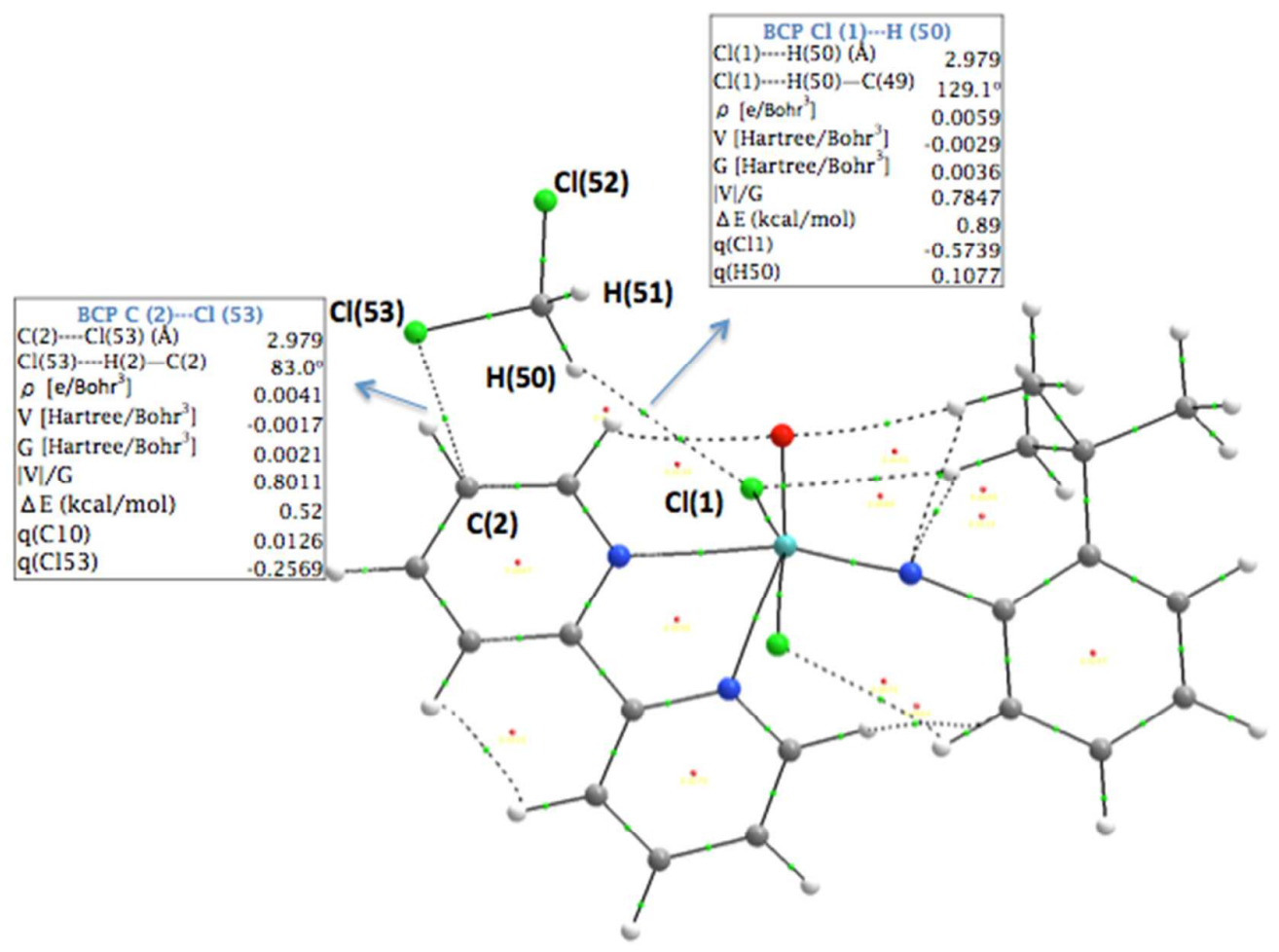




\section{Complex (b)}

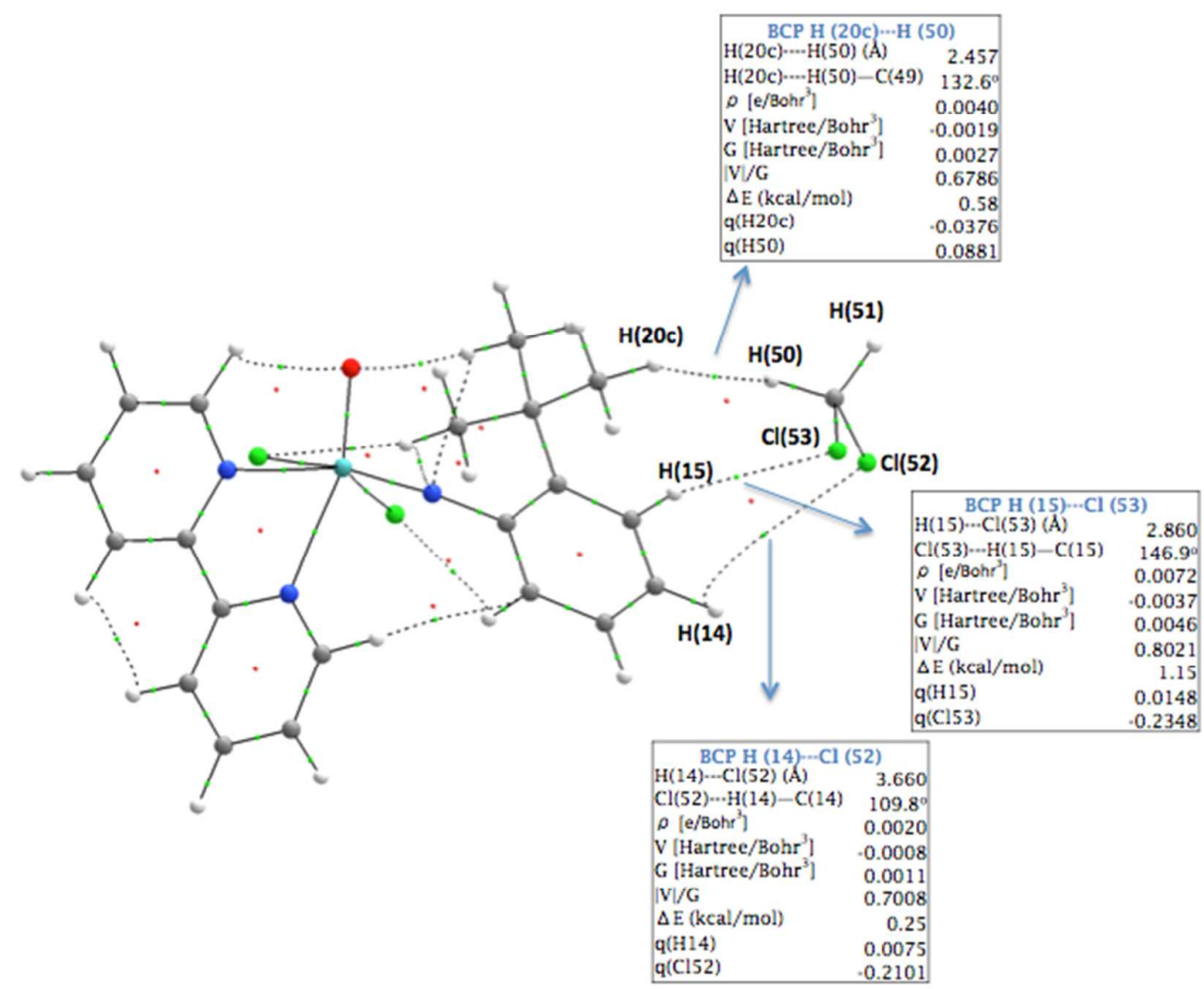

\section{Complex (c)}

\begin{tabular}{|c|c|}
\hline \multicolumn{2}{|c|}{ BCP C (13) - H (56) } \\
\hline$C(13) \cdots H(56)(A)$ & 3.641 \\
\hline $\mathrm{H}(56) \cdots \mathrm{H}(13)-\mathrm{C}(13)$ & $68.0^{\circ}$ \\
\hline$o\left[\mathrm{e} /\right.$ Bohr $\left.^{3}\right]$ & 0.0030 \\
\hline V [Hartree/Bohr ${ }^{3}$ ] & .0 .0012 \\
\hline G [Hartree/Bohr $\left.{ }^{3}\right]$ & 0.0017 \\
\hline $\mathrm{V} \mid / \mathrm{G}$ & 0.7059 \\
\hline$\Delta \mathrm{E}(\mathrm{kcal} / \mathrm{mol})$ & 0.38 \\
\hline$q(C 13)$ & -0.0121 \\
\hline$q(\mathrm{H} 56)$ & 0.0974 \\
\hline BCP C (11) $\cdots \mathrm{Cl}$ & \\
\hline$C(11) \ldots C l(57)(A)$ & 4.027 \\
\hline$C(11) \cdots C l(57)-C(54)$ & $97.8^{\circ}$ \\
\hline o [e/Bohr $]$ & 0.0027 \\
\hline V [Hartree/Bohr $\left.{ }^{3}\right]$ & -0.0012 \\
\hline G [Hartree/Bohr $\left.{ }^{3}\right]$ & 0.0034 \\
\hline$|\mathrm{V}| / \mathrm{G}$ & 0.7684 \\
\hline$\Delta \mathrm{E}(\mathrm{kcal} / \mathrm{mol})$ & 0.82 \\
\hline$q(C 11)$ & 0.4319 \\
\hline$q(C 157)$ & -0.2072 \\
\hline
\end{tabular}

BCP H $(19 \mathrm{~g})-\mathrm{Cl}$ (57)

H(19a)…Cl(57) (A $\quad 3.482$

$\mathrm{Cl}(57) \cdots(\mathrm{H} 19 \mathrm{a})-\mathrm{C}(20) \quad 129.5$

o [e/Bohr $\left.{ }^{3}\right]$

$\mathrm{V}$ [Hartree/Bohr ${ }^{3}$ ]

G [Hartree/Bohr ${ }^{3}$ ]

V $/ / \mathrm{G}$

0.002

( $\mathrm{cal} / \mathrm{mol})$

q(H19a)

0.25
0.0293

$\mathrm{q}(\mathrm{Cl} 57)$ -0.0293
.0 .2072

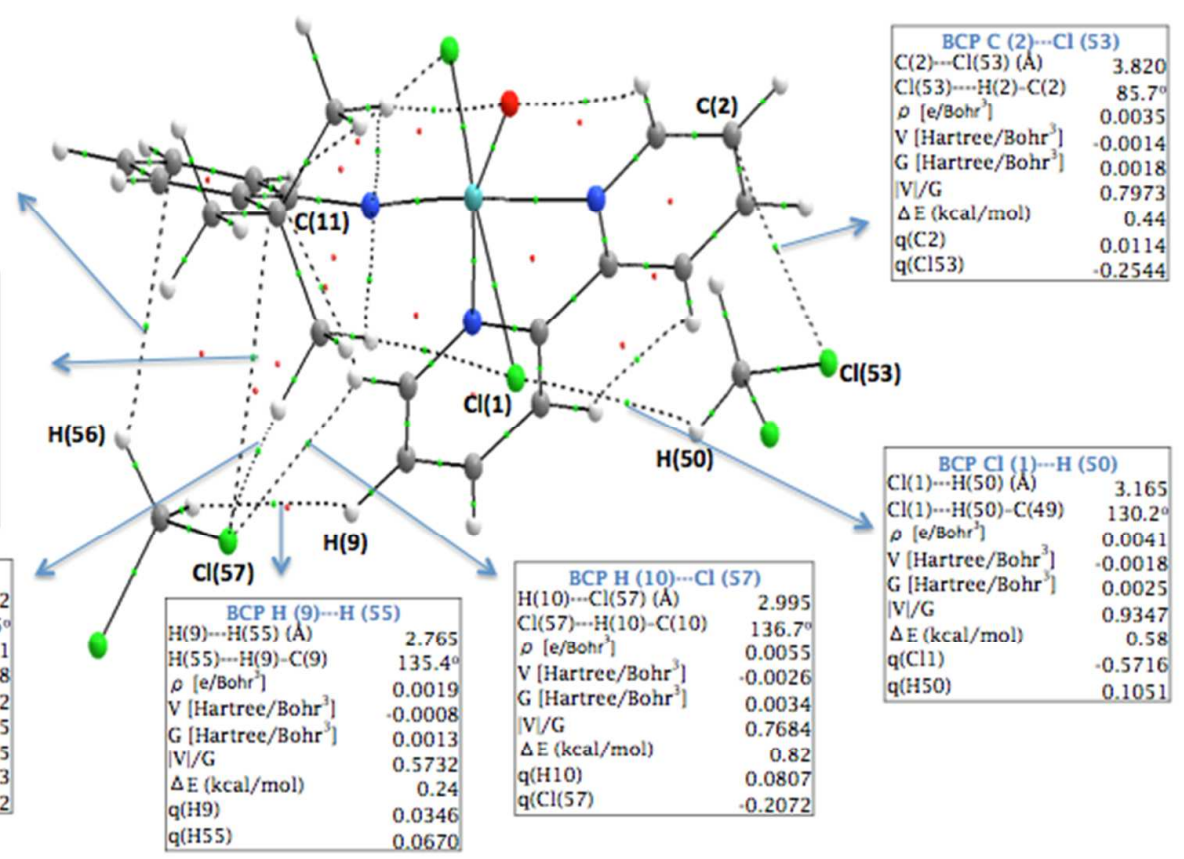




\section{Complex (d)}

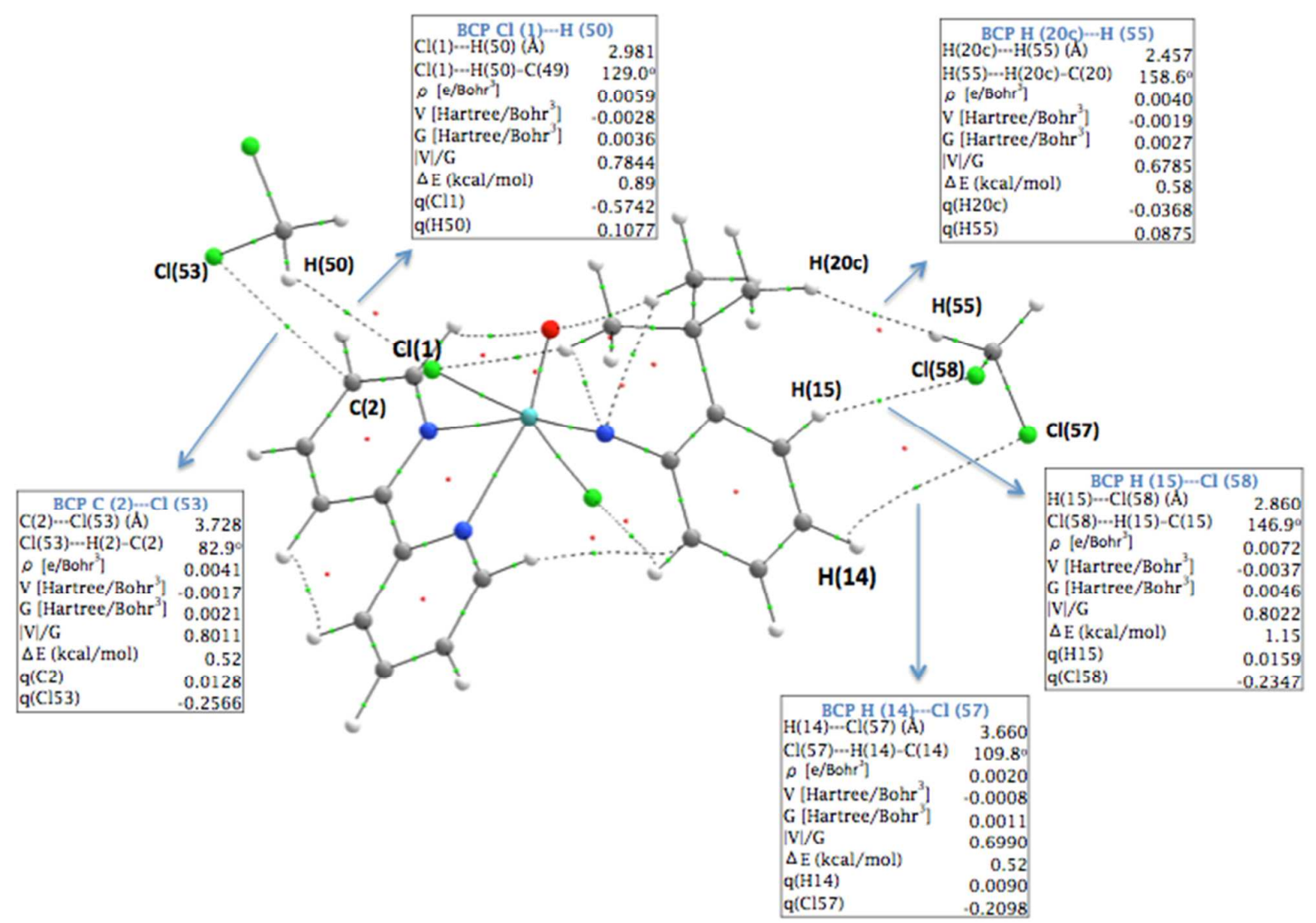

Supplementary Figure S14: Geometries of the complexes (a-d) containing $\mathrm{CH}_{2} \mathrm{Cl}_{2}$.

\section{Complex (a)}

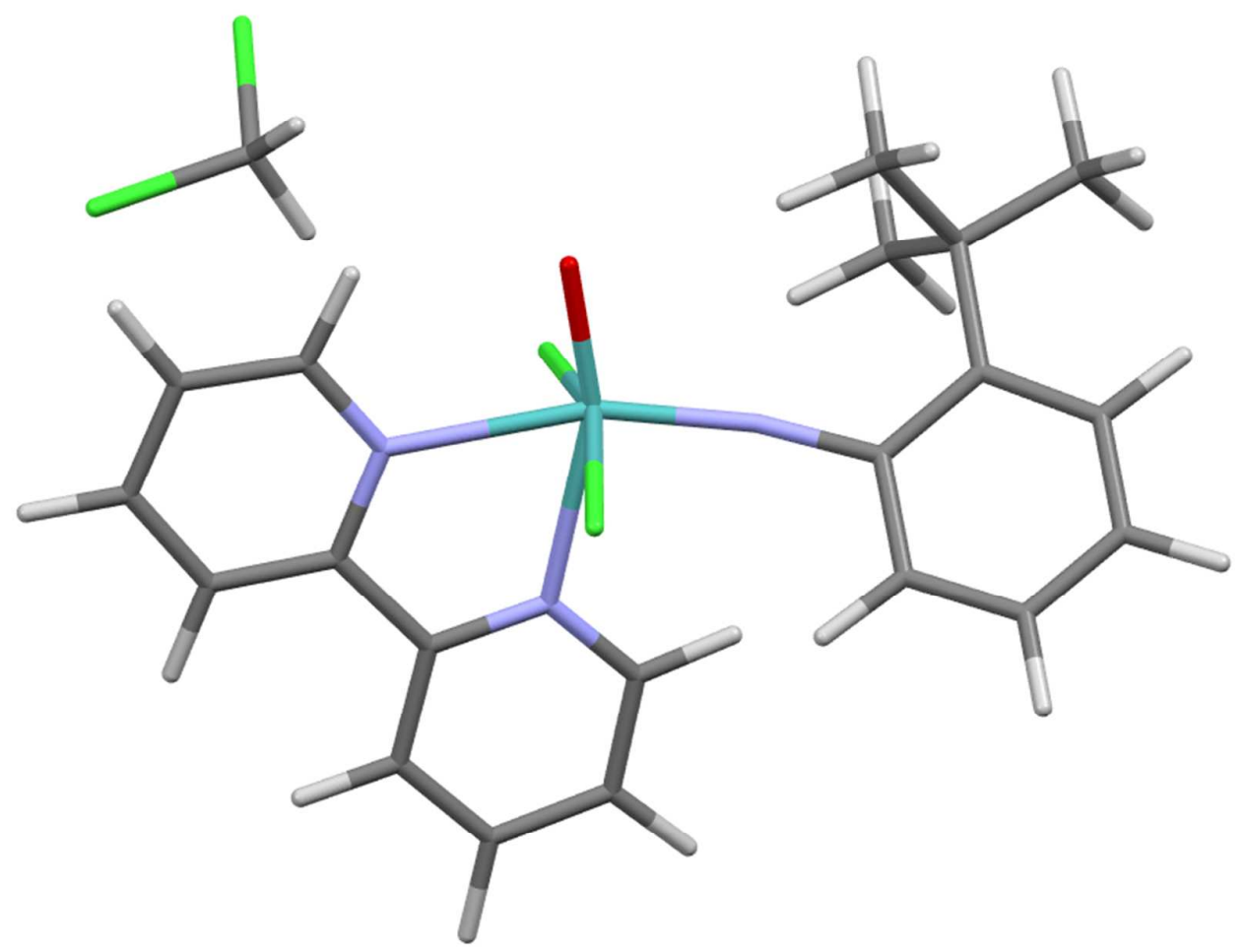


Complex (b)
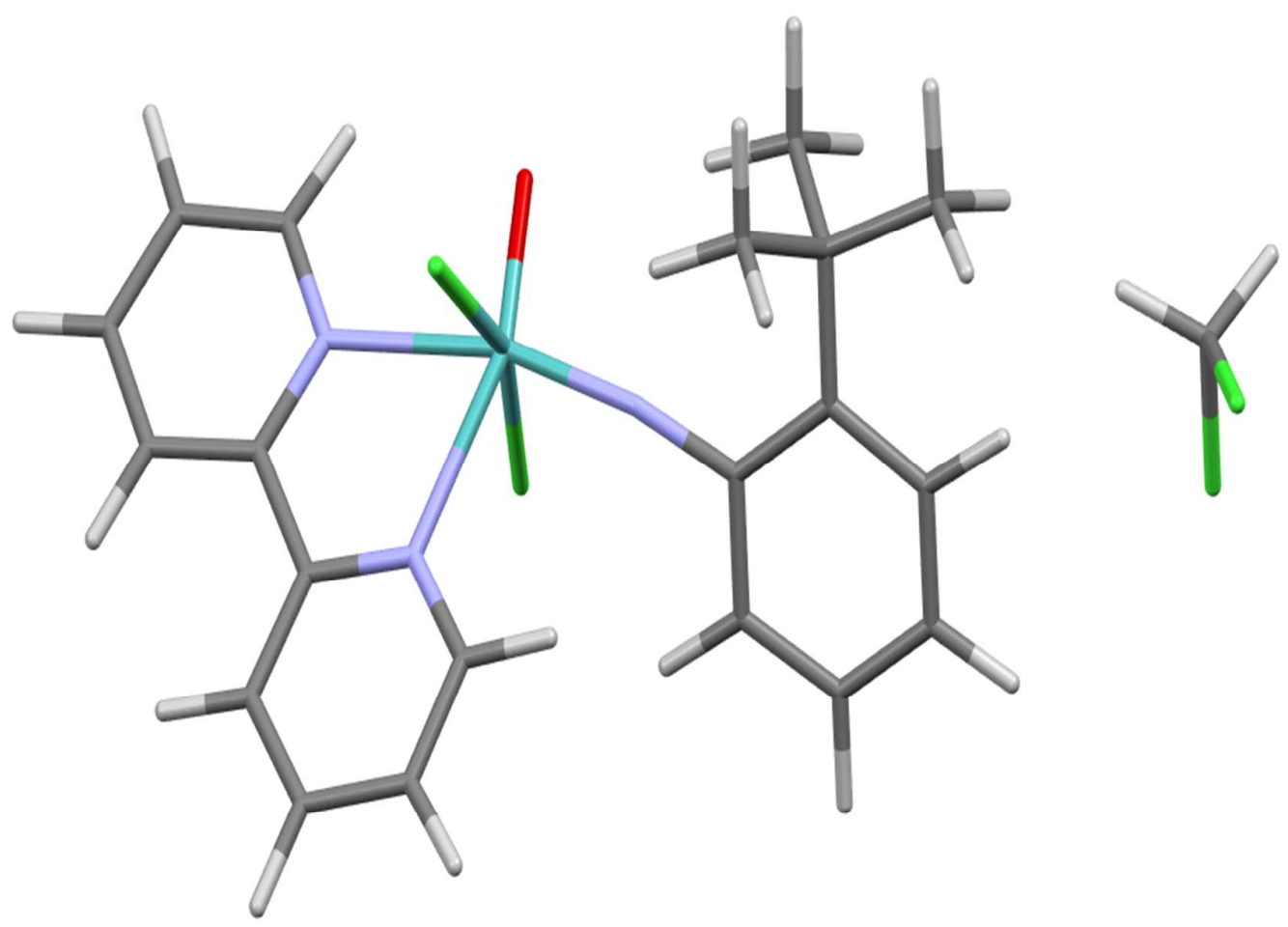

Complex (c)

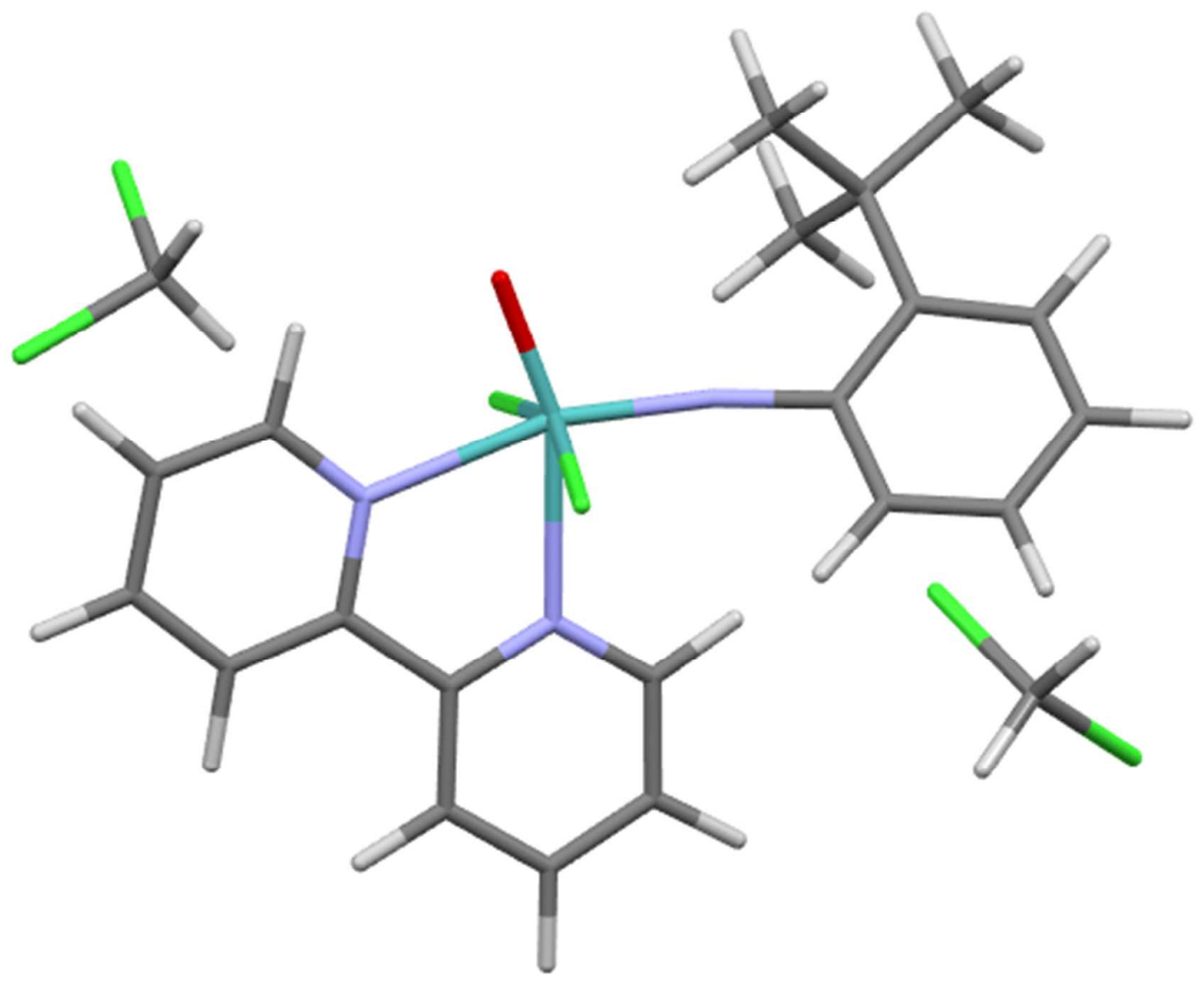




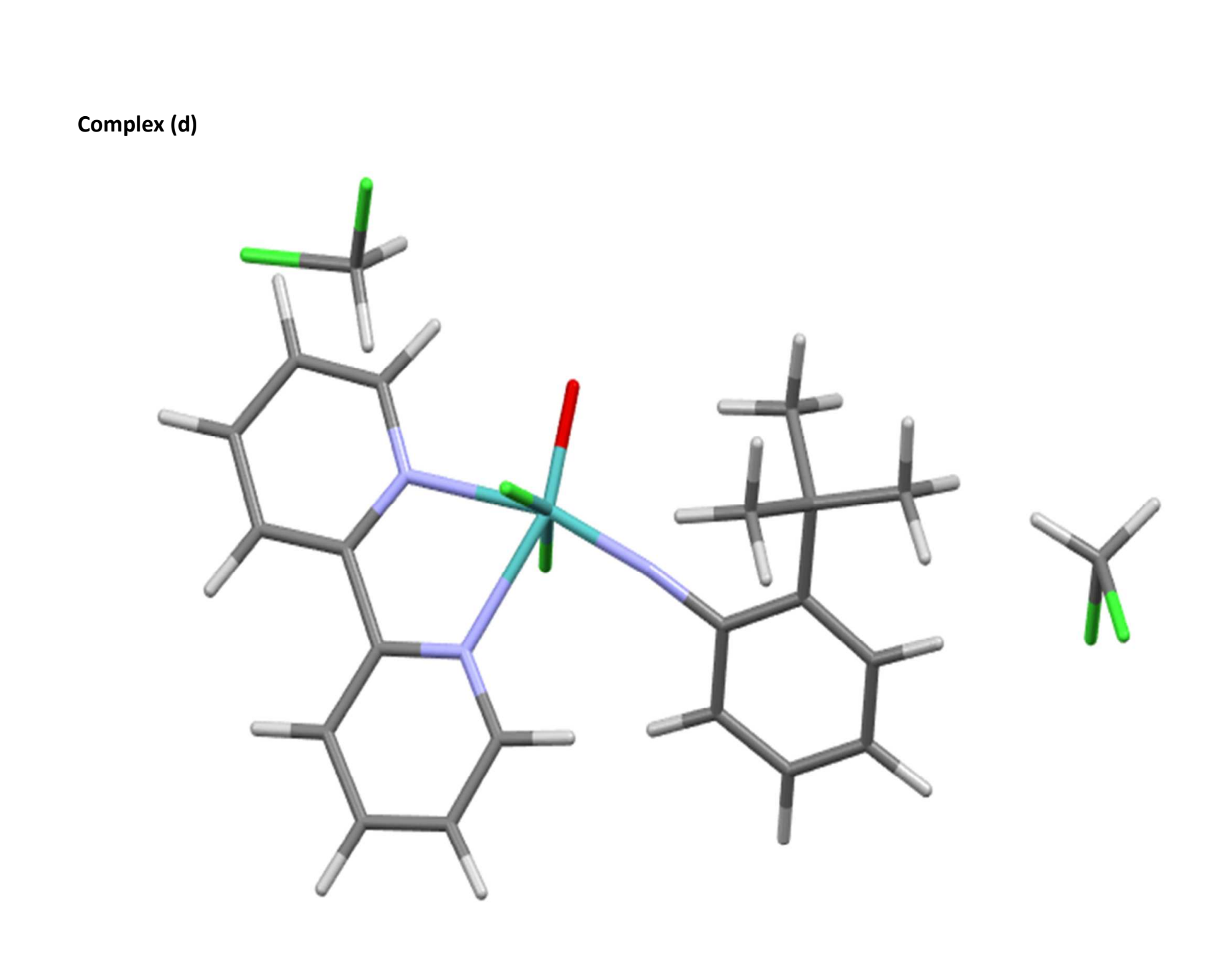

